\title{
Predicting modal characteristics of a cluster of cylinders in axial flow: from potential flow solutions to coupled CFD-CSM calculations
}

\author{
Jeroen De Ridder $^{\mathrm{a}}$, Joris Degroote ${ }^{\mathrm{a}}$, Katrien Van Tichelen ${ }^{\mathrm{b}}$, Jan Vierendeels ${ }^{\mathrm{a}}$ \\ ${ }^{a}$ Department of Flow, Heat and Combustion Mechanics, Faculty of Engineering and Architecture, Ghent University, Ghent, Belgium \\ ${ }^{b}$ Belgian Nuclear Research Centre, Mol, Belgium
}

\begin{abstract}
External fluid flow has a number of effects on the dynamics of a submersed structure: e.g., a solitary cylinder exposed to an external flow experiences added mass and damping due to the presence of the surrounding fluid. At high axial flow velocities relative to the stiffness of the cylinder, coupled instabilities such as flutter and divergence occur. Compared to a solitary cylinder, a cluster of cylinders also experiences inter-cylinder coupling: pressure perturbations in the fluid due to the movement or acceleration of one cylinder force another cylinder to move. Consequently, the different cylinders can move in organized patterns.

In this contribution, modal characteristics of a 7-rod bundle will be predicted by linear theory as well as by coupled CFD-CSM (Computational Fluid Dynamics - Computational Structure Mechanics) calculations. In the first part, fluid forces which lead to coupling of motion are computed with classical potential flow theory and URANS (Unsteady Reynolds-Averaged Navier-Stokes). Those forces are divided in a contribution in phase with the acceleration and a contribution in phase with the velocity of a cylinder. In the second part, modal characteristics of a 7 cylinder bundle are computed with coupled CFD-CSM simulations. The initial perturbations, which are required for the time-domain simulations come from a simplified structural model, with potential flow coupling between cylinders. The results are compared to linear theory. In the final part, approximations are proposed to predict upper and lower bounds of eigenfrequencies and damping, using calculations with only one cylinder.
\end{abstract}

Keywords: FSI, Axial flow, Turbulent flow, Cylinder arrays, Rod bundles

\section{Introduction}

Fuel rod bundles in nuclear reactors are continuously optimized in order to provide excellent heat transfer, while ensuring a low pressure drop and safe operation. As the resulting structure can be quite flexible, vibrations due to the interaction with the fluid flow might occur.

At low flow speed vibrations in rod bundles occur mainly due to turbulence in the flow. At intermediate specific flow speeds, resonance might occur with a flow instability. The most well-known example of this is the response of a flexibly mounted cylinder in cross-flow conditions to Von Karman vortices. If the flow direction is aligned with the cylinders in the bundle, Von Karman vortices are not present, but even then, in closely spaced rod bundles, other flow instabilities can occur (Meyer and Rehme, 1994). Additionally, in axial flow conditions vortices shed from supporting structures might trigger vibrations, as happens in grid spaced fuel bundles. At high flow speeds, positive feedback forces between structural displacement or velocity and fluid loading can cause instabilities such as divergence and flutter.

In order to predict the occurrence of any of these instabilities, the modal characteristics of the structure exposed to a fluid flow have to be known. Due to the external fluid flow, a solitary cylinder experiences added mass and damping. At high axial flow velocities relative to the stiffness of the cylinder, the influence of fluid forces on the modal characteristics becomes so large that coupled instabilities such as flutter and divergence occur. A cluster of cylinders in axial flow has additional complexity because it experiences inter-cylinder coupling as the pressure perturbation in the fluid due to the movement or acceleration of one cylinder forces another cylinder to move.

Due to the strong analogy with solitary cylinders, a small overview of literature on cylinders in axial flow is presented first. The earliest comprehensive models to predict the dynamics and the onset of instabilities of solitary 
cylinders in axial flow are the linear models by Païdoussis (1973) and Chen (1987). The fluid forces in these models are split in a viscous and an inviscid contribution. The inviscid contribution was derived by Lighthill (1960), while the viscous contribution is based on an empirical description (Taylor, 1952; Hoerner, 1965). Linear models based on this analysis are still widely used (Sakuma et al., 2008; Rinaldi and Païdoussis, 2012; Kheiri and Païdoussis, 2015). Overall, they provide a good description of the natural frequency of the cylinder in the stable regime, while damping can be tuned by changing viscous coefficients. Regarding instabilities, they provide a good estimation for the onset of divergence. However, the prediction of flutter instabilities for a clamped-clamped cylinder is very sensitive to friction coefficients (Païdoussis, 1973). Ersdal and Faltinsen (2006) and Divaret et al. (2014) investigated those friction forces perpendicular to the cylinder experimentally. One of the major findings of the latter work is that at low angles of attack, the normal friction force is a linear function of the angle of attack, while at high angles of attack, it is a quadratic function.

In order to examine the post-critical behavior and to verify the validity of linear theory, a weakly non-linear theory was developed, first for cantilevered cylinders in a three-article publication (Lopes et al. (2002); Païdoussis et al. (2002); Semler et al. (2002)). A similar analysis was later performed for very long cylinders by de Langre et al. (2007). Dynamics for a vanishing stiffness can be found in Triantafyllou (1985). The weakly non-linear theory (Semler et al. (2002)) was later applied to pinned-pinned and clamped-clamped cylinders (Modarres-Sadeghi et al. (2007)). This theory showed, just like the linear theory, the existence of a flutter motion. An additional series of experiments, performed by Modarres-Sadeghi et al. (2008), was in qualitative agreement with the non-linear theory. However, there is a difference of $40 \%$ in the threshold at which flutter is predicted and the fluttering motion was also observed around the neutral axis and not around a buckled state, as calculated by weakly non-linear theory. To increase the accuracy of the predictions, the viscous coefficients were computed numerically in Jamal et al. (2014).

In a fully non-linear coupled CFD-CSM (Computational Fluid Dynamics - Computational Structure Mechanics) simulation, we investigated the modal characteristics of a single cylinder in axial flow, minimizing the amount of required empirical input (De Ridder et al., 2013). This analysis was subsequently used to predict fluidelastic instabilities of a flexible cylinder in axial flow, which was more accurate than weakly non-linear theory (De Ridder et al., 2015).

The main difference between a cluster of cylinders and a solitary one is that there is not a single added mass per cylinder. Instead, pressure and velocity perturbations due to the movement of one cylinder create forces on neighboring cylinders. The resulting added mass matrix was first derived using potential flow theory by Chen (1987); Païdoussis and Suss (1977); Païdoussis (1979). The viscous coupling matrix was first presented by Lin (1987), as an extension of the work by Chen (1987) on two concentric cylinders. Unfortunately, this analysis is only valid for laminar flow conditions.

In this paper, the modal characteristics of 7-cylinder cluster in turbulent axial flow are computed using fully coupled CFD-CSM calculations. This geometry is a simplified representation of a true fuel assembly. In the first part of the paper, the methodology section is explained and the fluid coupling forces are computed by URANS (Unsteady Reynolds-Averaged Navier-Stokes) and potential flow. In the second part, the characteristics are computed by fully coupled simulations, which use the linear theory results as a precursor step. In the final part approximations are proposed for the upper and lower bounds of eigenfrequencies and damping, using calculations with only one cylinder.

\section{Methodology}

The methodology section contains details on the different types of simulations used throughout this paper. It starts with linear theory, followed by a small paragraph on CFD simulations with forced motion and a part on coupled CFD-CSM simulations for the prediction of modal characteristics. It ends with a description of the geometry and the numerical settings.

\subsection{Linear Theory}

The results from the coupled simulations will be compared to those predicted by linear theory. The linear approximation is a simplified version of the derivation by Païdoussis and Suss (1977) and Païdoussis (1979).

As the flexible cylinder is long and slender, the Euler-Bernoulli beam theory is applicable, to which the fluid forces are added

$$
E I \frac{\partial^{4} u_{i}}{\partial z^{4}}+F_{N I i}^{x}+F_{N V i}^{x}-F_{L i}^{x} \frac{\partial u_{i}}{\partial z}-\frac{\partial}{\partial z}(T+p A) \frac{\partial u_{i}}{\partial z}+m \frac{\partial^{2} u_{i}}{\partial t^{2}}=0
$$


In this equation, $E$ is the Young's modulus of elasticity, $I$ is the area moment, $u_{i}$ the displacement of cylinder $i$ in $x-$ direction and $z$ stands for the axial direction. $F_{N I i}^{x}, F_{N V i}^{x}$ and $F_{L i}^{x}$ are the hydrodynamic inviscid normal, viscous normal and tangential force (per unit length) respectively. $m$ is the linear mass density of the cylinder, which is non-uniform in this case. $T$ is the axial tension, $p$ the pressure in the bundle and $A$ the cross-sectional area of the cylinder. In Equation 1, the gravity term, external tension and pressurization are ignored, as the focus of this paper is on studying hydrodynamic coupling. A similar equation can be written in the y-direction.

The hydrodynamic normal inviscid force is, in this linear theory, determined from a potential flow analysis, which is provided in Appendix $\mathrm{A}$ and which results in

$$
\begin{aligned}
& F_{N I i}^{x}=-\rho_{f} \pi R_{i}^{2} \sum_{l=1}^{K}\left(\left(\alpha_{1 i l}+\gamma_{1 i l}\right) \frac{\mathrm{D}^{2} u_{l}}{\mathrm{D} t^{2}}+\left(a_{1 i l}+c_{1 i l}\right) \frac{\mathrm{D}^{2} v_{l}}{\mathrm{D} t^{2}}\right), \\
& F_{N I i}^{y}=-\rho_{f} \pi R_{i}^{2} \sum_{l=1}^{K}\left(\left(\beta_{1 i l}+\delta_{1 i l}\right) \frac{\mathrm{D}^{2} u_{l}}{\mathrm{D} t^{2}}+\left(b_{1 i l}+d_{1 i l}\right) \frac{\mathrm{D}^{2} v_{l}}{\mathrm{D} t^{2}}\right) .
\end{aligned}
$$

In this equation $\frac{D}{D t}=\frac{\partial}{\partial t}+V \frac{\partial}{\partial z}$, with $V$ the mean axial flow velocity. $\rho_{f}$ is the fluid density, $R_{i}$ the radius of cylinder $i, K$ the number of cylinders and $\alpha_{1 i l}-d_{1 i l}$ are coefficients which can be found from Equation A.20. Please note the small $u$ and $v$ are used for cylinder displacement in $\mathrm{x}$ - and y-direction, while capital $V$ denotes flow velocity in axial (z-) and transversal (x-,y-) direction.

In the approach followed by Païdoussis and Suss (1977) the viscous normal force results from an empirical relation

$$
F_{N V i}^{x}=\rho_{f} R_{i} V^{2} C_{N} \alpha_{x}
$$

In this equation $\alpha_{x}$ is the flow incidence angle and $C_{N}$ the normal force coefficient. In the paper by Païdoussis and Suss (1977) an additional term is included for the correct behavior at zero flow velocity. As this term is much smaller than the one in Equation 4 for turbulent flows, it is omitted here. The incidence angle of the flow (rad) is given by

$$
\alpha_{x}=\arctan \left(\frac{\partial u_{i}}{\partial z}\right)+\arctan \left(\frac{\partial u_{i}}{\partial t}-V_{x i}\right) \frac{1}{V} \approx \frac{\partial u_{i}}{\partial z}+\left(\frac{\partial u_{i}}{\partial t}-V_{x i}\right) \frac{1}{V}
$$

The $V_{x i}$-term represents the average transverse flow velocity induced by the motion or presence of neighboring cylinders. This velocity is also computed from the potential flow analysis in Appendix C.

The longitudinal force $F_{L i}^{x}$ is evaluated as

$$
F_{L i}^{x}=\rho_{f} R_{i} V^{2} C_{f} \frac{D_{h}}{D}
$$

with $D_{h}$ the hydraulic diameter. Note that buoyancy is ignored in this force as the effect of this longitudinal force is relatively small. If the assembly is placed horizontally, buoyancy will lead to a static deflection. It hence influences the dynamics only indirectly. If the assembly is mounted vertically, buoyancy forces might alter the dynamics of the cylinders. However, this depends on the boundary conditions as well as on the relative strength of buoyancy compared to stiffness.

The effect of tension and pressure drop is taken into account by

$$
\begin{aligned}
\frac{\partial}{\partial z}\left((T+p A) \frac{\partial u_{i}}{\partial z}\right) & =\frac{\partial}{\partial z}\left(\left(\rho_{f} R_{i} V^{2} C_{f}-\frac{\partial p}{\partial z} A\right)(L-z) \frac{\partial u_{i}}{\partial z}\right) \\
& =\rho_{f} R_{i} V^{2} C_{f}\left(1+\frac{D_{h}}{D}\right) \frac{\partial}{\partial z}\left((L-z) \frac{\partial u_{i}}{\partial z}\right)
\end{aligned}
$$

This includes the direct effect of friction and the pressure drop effect, but buoyancy and base drag are ignored in this paper.

Equation 1 is solved with a Galerkin-procedure. The basis functions $\phi_{i}(z)$ are the normalized $\left(\int_{0}^{L} \phi_{i}(z) \phi_{j}(z) \mathrm{d} z=\right.$ $\delta_{i j}$ ) eigenmodes of a clamped-pinned beam. These boundary conditions are used in all the cases in this paper. Alternatively, the boundary conditions could be implemented as a constraint with a Lagrangian multiplier. Note that the 


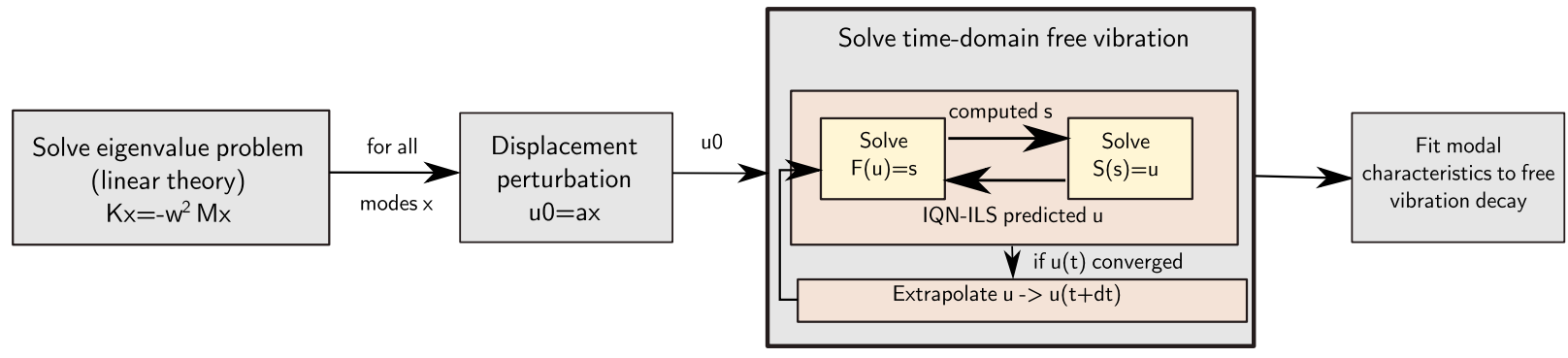

Figure 1: Flow chart of the coupled CFD-CSM simulation procedure ( $x$ is the eigenmode shape according to linear theory, a a pre-defined amplitude, $\mathrm{F}$ and $\mathrm{S}$ represent (the flow and structural solver), $\mathrm{u}$ is the interface displacement and $\mathrm{s}$ the traction on the interface).

basis functions are in $\mathrm{x}$ - and $\mathrm{y}$-direction and per cylinder. The displacement is subsequently written as $\sum_{i} q_{i}(t) \phi_{i}(z)$. Equation 1 is multiplied with $\phi_{j}(z)$ and integrated over $z=0 . . L$, which leads to

$$
M \ddot{q}+C \dot{q}+K q=0
$$

with q a vector containing the time-dependent coefficients of the Galerkin expansion. The eigenvalues and eigenmodes are then found by replacing the previous system with

$$
\left[\begin{array}{ll}
0 & I \\
K & C
\end{array}\right]\left[\begin{array}{c}
q \\
\dot{q}
\end{array}\right]=-\left[\begin{array}{cc}
-I & 0 \\
0 & M
\end{array}\right]\left[\begin{array}{c}
\dot{q} \\
\ddot{q}
\end{array}\right] .
$$

This leads to a generalized eigenvalue problem.

\subsection{CFD simulations with and without imposed motion of the central cylinder}

In section 3.1, the flow in a bundle of rigid and stationary cylinders is investigated with RANS (Reynolds-Averaged Navier-Stokes) simulations. In the subsequent section, the induced forces due to transverse movement of a cylinder will be computed using potential flow theory and URANS simulations. In the URANS simulations, the central cylinder of the cylinder cluster (see section 2.4) undergoes a sinusoidal translational motion. The resulting fluid forces are monitored on all cylinders. These forces are subsequently decomposed in forces in phase with the displacement (or acceleration) and in phase with the velocity.

\subsection{Coupled CFD-CSM simulations to predict modal characteristics}

The modal characteristics are extracted from numerical simulations in a similar way as done earlier for a single cylinder (De Ridder et al., 2013). The flow chart in Figure 1 summarizes the method. It starts with an initial estimation of mode shapes and frequencies. In this case, an eigenvalue system is created based on linear theory. As it is only an initial guess, the system in Section 2.1 is simplified by omitting all damping terms. The resulting modes serve as perturbation for coupled CFD-CSM or fluid-structure interaction (FSI) simulations. The eigenfrequencies are used to select an appropriate time step $\left(\Delta t \approx\left(2 \pi / \omega_{n, \text { est }}\right) / 200\right)$, with $\omega_{n, \text { est }}$ the estimated natural frequency. This time step is based on the analysis in (De Ridder et al., 2013).

As the motion of the cylinders in the bundle is coupled by the liquid in between them, every beam mode splits into $2 \mathrm{~K}$ modes, with $\mathrm{K}$ the number of cylinders. Every coupled mode is subsequently used as the initial displacement perturbation in an FSI-simulation, which computes the free vibration decay of that perturbation. The initial displacements amplitude was typically $0.1 \mathrm{~mm}$. In the time-dependent simulations, a strong coupling is used, ensuring a converged solution during every time step. To maintain a stable numerical scheme, even if the density of the fluid is comparable to the density of the solid, the IQN-ILS-algorithm is used (Degroote et al., 2009).

In the final step of the flow chart, a modal expansion is fitted to the computed center line displacement of every cylinder i:

$$
u_{c l, i} \approx \sum_{j=1}^{N} a_{i, j}(z) \exp ^{-\zeta_{j} \omega_{n, j} t} \cos \left(\sqrt{1-\zeta_{j}^{2}} \omega_{n, j} t+\phi_{i, j}(z)\right)
$$




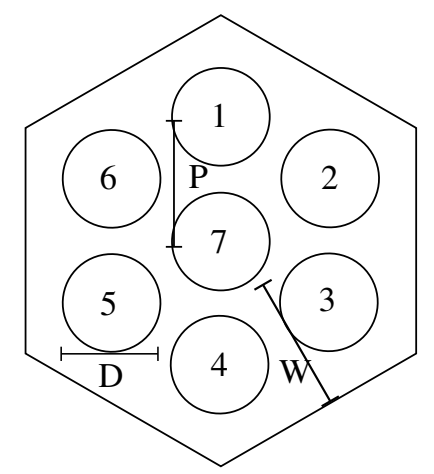

(a) Sketch of a cross-section with indication of cylinder labels and main dimensions (P:pitch, D:diameter, W:width).

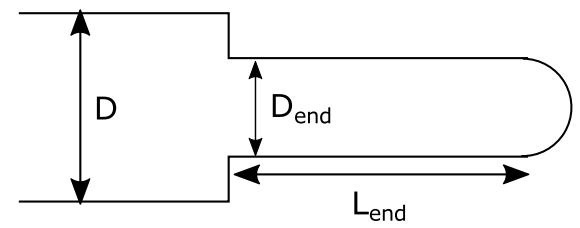

(b) Sketch of the end piece with main dimensions.
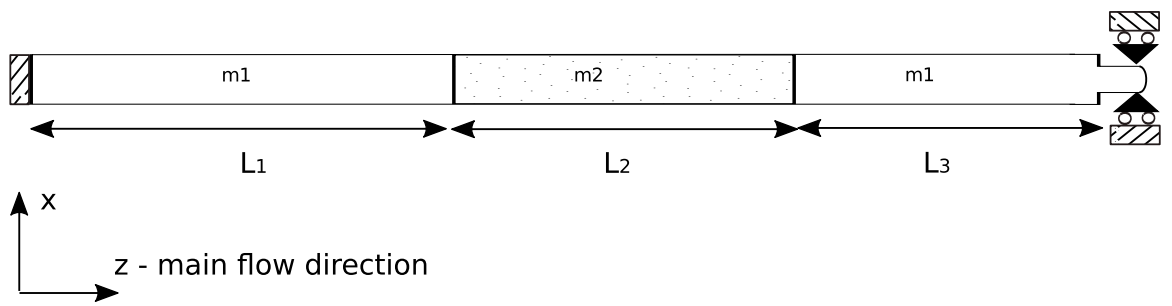

(c) Sketch of cylinder with main dimensions, structural boundary conditions and mass distribution.

Figure 2: Geometry of the 7-rod bundle

in which $a_{i, j}(z)$ is the amplitude of mode $\mathrm{j}$ on cylinder $\mathrm{i}$ at height $\mathrm{z}, \zeta_{j}$ is the modal damping ratio, $\omega_{n, j}$ the natural frequency, $\phi_{i, j}(z)$ the phase difference and $N$ the number of required modes. In the cases investigated in this paper, it was sufficient to use one mode in the summation above. The phase $\phi_{i, j}(z)$ was also uniform over the length of the cylinder. Note that one mode spans multiple cylinders, which means that all cylinders can move in different directions, but at the same frequency and damping.

\subsection{Geometry and parameters}

The geometry under consideration is a cluster of 7 cylinders, which is depicted in Figure 2a. The diameter D of the cylinders, the pitch P between the cylinder centra as well as the distance $\mathrm{W}$ are provided in Table 1. Both the linear theory and the numerical simulations are applied over the total length of the cylinders. In the potential flow analysis a circular exterior confinement is used, with a radius of $12.95 \mathrm{~mm}$, such that the cross-sectional area of the circle is the same as for the hexagon. The cylinders are clamped upstream, corresponding to a rigid support which prevents coupling between the cylinder vibrations through the support at that location. The downstream end is simply supported, but free to slide in axial direction. The structural parameters are given in Table 2. The middle part of the cylinder (from $\mathrm{z}=0.6275 \mathrm{~m}$ to $\mathrm{z}=1.2275 \mathrm{~m}$ ) has a different solid density $\left(\mathrm{m}_{2}\right)$ than the outer parts $\left(\mathrm{m}_{1}\right)$. The fluid properties of the incompressible liquid lead-bismuth eutectic are listed in Table 2. Under standard conditions a bulk inlet velocity of $2 \mathrm{~m} / \mathrm{s}$ is imposed, with a turbulence intensity of $5 \%$ and a turbulence length scale of $1 \mathrm{~mm}$. The outlet is kept at constant pressure and no-slip boundary conditions are applied at the walls.

The grid is based on the grid sensitivity study by De Ridder et al. (2013). The fluid grid in between the different cylinders consists of 10 cells normal to the wall, 36 cells along the circumference of the cylinders and 200 cells in axial direction. It is displayed along with the structural grid in Figure 3. All discretization schemes are second-order accurate, both in time and in space. As the flow is turbulent and changing in time due to the cylinder motion, Unsteady 
Table 1: Geometrical properties. The geometry is schematically depicted in Figure 2.

\begin{tabular}{cccc|cc|ccc}
\hline \hline & Geometrical properties & \multicolumn{4}{c}{ Details of end piece } & \multicolumn{3}{c}{ Lengths of different sections } \\
$\mathrm{D}$ & $\mathrm{P}$ & $\mathrm{W}$ & $\mathrm{L}$ & $\mathrm{D}_{\text {end }}$ & $\mathrm{L}_{\text {end }}$ & $\mathrm{L}_{1}$ & $\mathrm{~L}_{2}$ & $\mathrm{~L}_{3}$ \\
\hline $6.54 \mathrm{~mm}$ & $8.40 \mathrm{~mm}$ & $8.40 \mathrm{~mm}$ & $1.4 \mathrm{~m}$ & $4.5 \mathrm{~mm}$ & $7 \mathrm{~mm}$ & $0.6275 \mathrm{~m}$ & $0.6 \mathrm{~m}$ & $0.1725 \mathrm{~m}$ \\
\hline \hline
\end{tabular}

Table 2: Fluid and structural properties and flow boundary conditions ( $\rho_{f}$ : fluid density, $\mu$ : dynamic viscosity, $\mathrm{V}_{\mathrm{i}}$ : bulk flow velocity, TI: turbulence intensity, TLS: turbulence length scale).

\begin{tabular}{cc|ccc|ccc}
\hline \hline \multicolumn{2}{c}{ Fluid properties } & \multicolumn{3}{c}{ Flow properties } & \multicolumn{3}{c}{ Structural properties } \\
$\rho_{f}$ & $\mu$ & $\mathrm{V}_{\mathrm{i}}$ & TI & TLS & EI & $\mathrm{m}_{1}$ & $\mathrm{~m}_{2}$ \\
\hline $10290 \mathrm{~kg} / \mathrm{m}^{3}$ & $0.0017 \mathrm{~Pa} . \mathrm{s}$ & $2 \mathrm{~m} / \mathrm{s}$ & $5 \%$ & $1 \mathrm{~mm}$ & $10.3 \mathrm{Nm}^{2}$ & $0.0664 \mathrm{~kg} / \mathrm{m}$ & $0.331 \mathrm{~kg} / \mathrm{m}$ \\
\hline \hline
\end{tabular}

Reynolds-Averaged Navier Stokes (URANS) simulations are used, with the k- $\omega$ SST model by Menter (1994). It was shown that this model is capable of predicting the forces with reasonable accuracy (Divaret et al., 2014) and is hence sufficient for the present simulations.

\section{Fluid forces}

\subsection{Fluid flow through steady geometry}

The axial velocity is not uniform through the bundle, as assumed in linear theory. The difference in axial velocity, normalized by the bulk velocity is visualized in Figure 4a. It shows that the axial velocity is highest in edge subchannels (up to 1.25 the bulk velocity), followed by interior subchannels. In the gap and corner regions, the flow velocity is the lowest with a maximal velocity up to the bulk velocity.

These differences in axial flow velocity result from the difference in hydraulic diameter between e.g. an interior subchannel and the gap region as shown by the following formulas. The reasoning starts by assuming that the pressure drop in axial direction is equal in a subchannel or a gap region. Note that this does not mean that the pressure has to be uniform in a cross section. The reasoning goes on by expressing the pressure drop as a function of wall shear stress $\tau_{w}$, which leads to a ratio of bulk velocity in the subchannel over velocity in the gap region.

$$
\begin{aligned}
& \Delta p_{\text {subchannel }}=\Delta p_{\text {gap }} \\
& \Rightarrow\left(\tau_{w} C / A\right)_{\text {subchannel }}=\left(\tau_{w} C / A\right)_{\text {gap }} \\
& \Rightarrow\left(0.5 c_{f} \rho V^{2} \frac{\pi D / 2}{\sqrt{3} / 4 P^{2}-\pi D^{2} / 8}\right)_{\text {subchannel }}=\left(0.5 c_{f} \rho V^{2} \frac{2}{P-D}\right)_{\text {gap }} \\
& \Rightarrow \frac{V_{\text {subchannel }}}{V_{\text {gap }}}=\sqrt{\frac{\sqrt{3} / 2 P^{2}-\pi D^{2} / 4}{(P-D) \pi D / 2}}
\end{aligned}
$$

In these equations, $C$ is the wetted perimeter and $A$ the through-flow area of the gap or subchannel region. The estimation provided by Equation 15 is a very rough estimation, as the friction constant $c_{f}$ is assumed to be constant in the entire domain, which is not the case (Cheng and Todreas, 1986). With a P/D-ratio of 1.28 the velocity ratio is 1.2, which is an overprediction of the 1.14 in Figure $4 \mathrm{a}$.

At the end of the bundle, the different jets from the subchannels combine into a larger channel flow. The turning of the jets requires a transverse pressure gradient, which can be seen on the end pieces in Figure $4 \mathrm{~b}$. Consequently, a net force is exerted on the end pieces of the cylinders.

In very tightly packed rod bundles, a flow instability similar to a Kelvin-Helmholtz instability has been observed (Hooper and Rehme, 1984; Rehme, 1992; Möller, 1991; Meyer and Rehme, 1994). However, the P/D-ratio of 1.28 is too high to observe any such instabilities. 


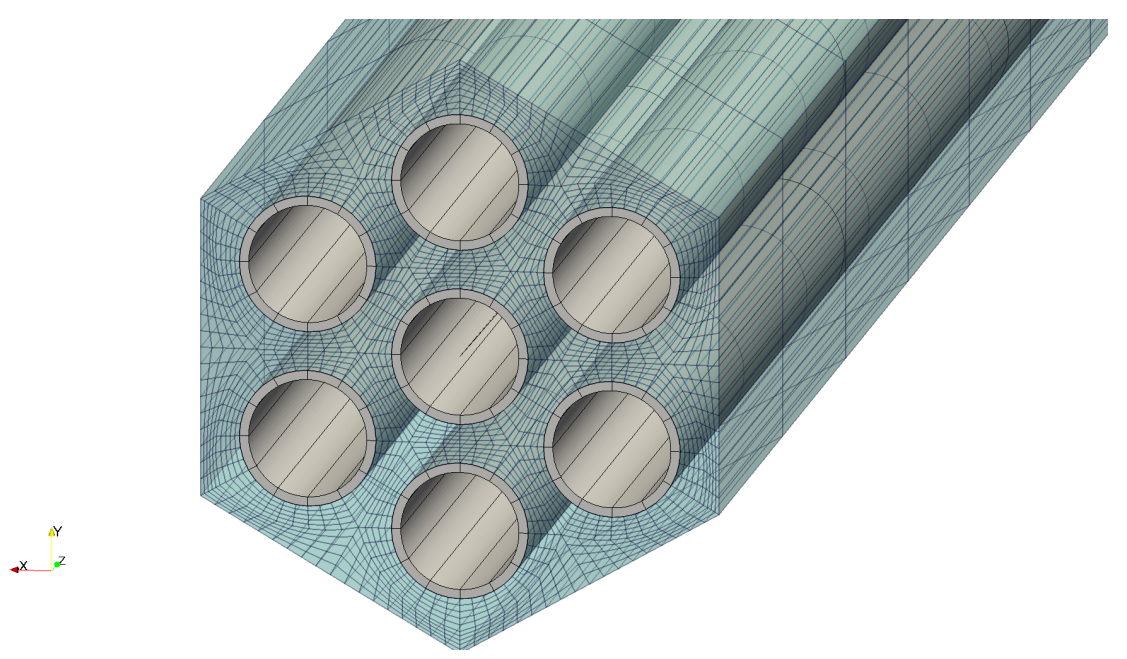

Figure 3: Fluid grid (blue) and structural grid (gray) of the 7-rod bundle.
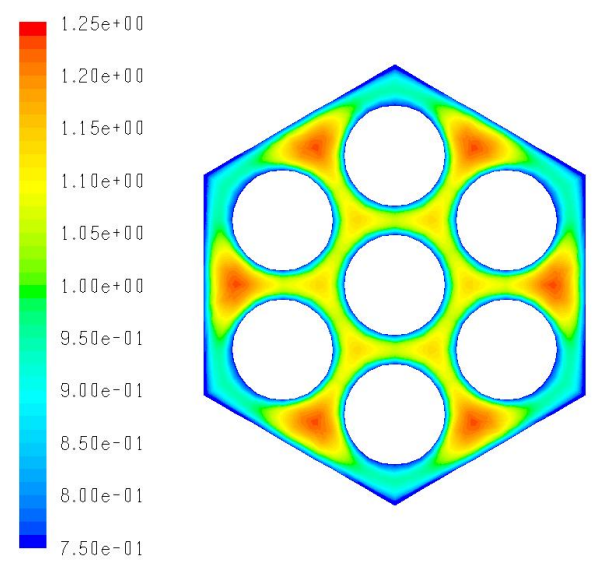

(a) Relative axial velocity with respect to the bulk flow velocity $\left(V / V_{i}\right)$ in the bundle, showing the velocity difference in interior, edge, corner subchannels and gap regions.
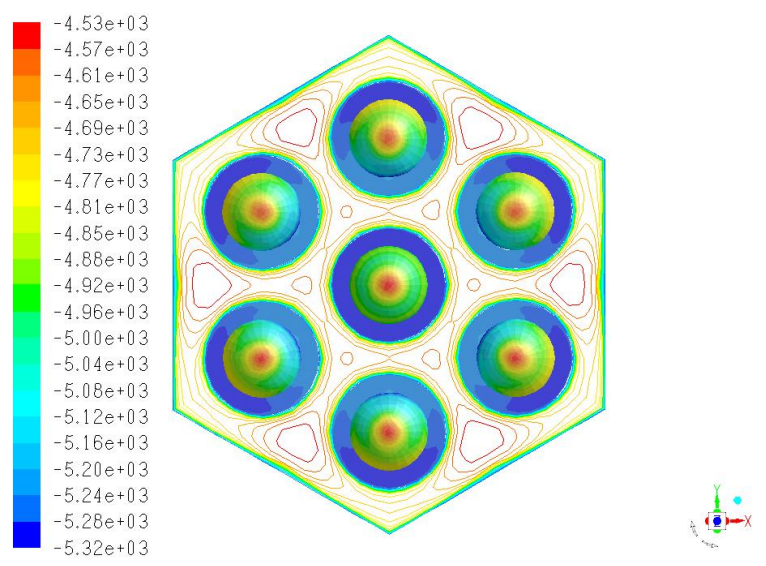

(b) Pressure contours $(\mathrm{Pa})$ on the end-pieces and velocity contours in the bundles.

Figure 4: Non-uniform steady flow through a 7-rod bundle 


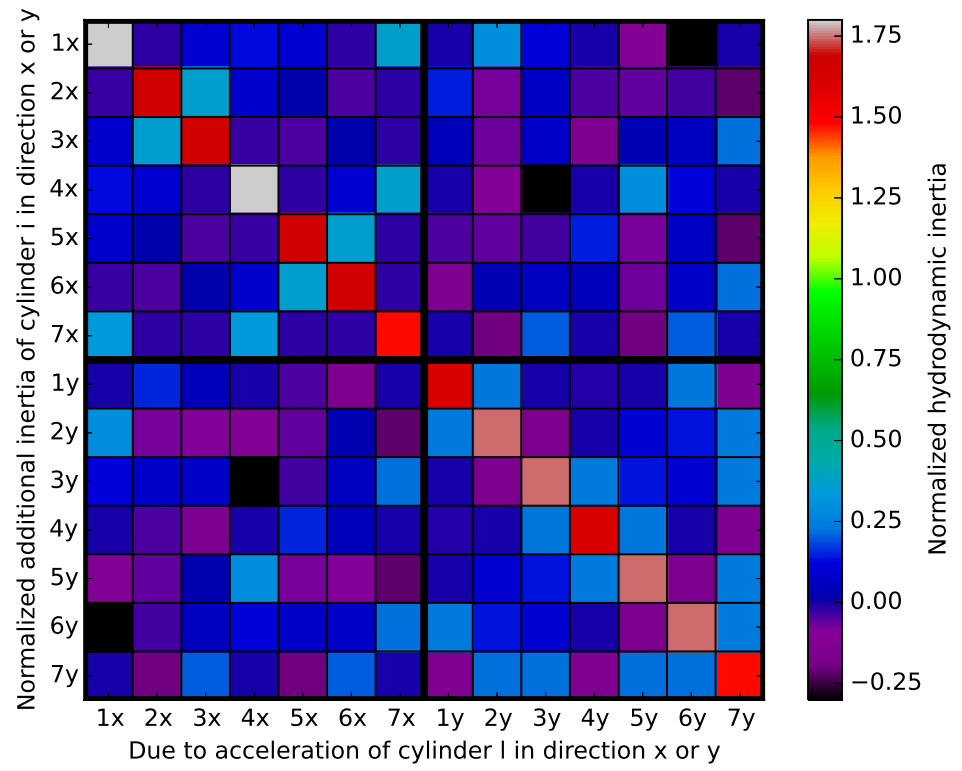

Figure 5: Inertial coupling between multiple cylinders, as predicted by potential flow theory.

\subsection{Forces on oscillating cylinders}

In the linear theory, the fluid forces are split into an inviscid and a viscous part. The inviscid forces result from a potential flow analysis, which is given in Appendix B. These viscous forces are given by an empirical correlation, which needs the average relative flow velocity, transverse to the cylinder. The transverse velocity in the linear theory in this paper is computed from the same potential, as shown in Appendix C. In this section it is verified to which extent those fluid forces are similar to those obtained from URANS simulations. In these URANS simulations, one cylinder is undergoing a sinusoidal rigid body motion, as explained in section 2.2 . The resulting fluid forces, which are in phase with the rigid body acceleration, correspond to the inviscid forces while those that are in phase with velocity correspond to viscous forces. If the displacement of the cylinder is given by $u=\cos (\omega t)$, the two components are found by minimizing

$$
\|F-a \cos (\omega t)-b \sin (\omega t)\|_{2} .
$$

The a-component is in phase with the cylinder's acceleration, while the b-component is in phase with the velocity.

\subsubsection{Inviscid forces}

The motion is imposed uniformly along the length of the bundle. Consequently, the resulting inviscid forces in Equations 2 and 3 contain only terms proportional to the acceleration of cylinders. Equations 2 and 3 can be rewritten in matrix form as $F=M_{a} \frac{\partial^{2} u}{\partial t^{2}}$, where $F$ is the vector containing the x- and y-force on all cylinders and $u$ a vector containing the displacement of all cylinders. As this force is a linear combination of accelerations, the $M_{a}$-matrix is the added mass (or hydrodynamic mass) matrix.

Figure 5 is a graphical representation of the added mass matrix, computed by potential flow theory. The hydrodynamic inertia of cylinder $i$ due to acceleration of cylinder 1 follows from the color in the grid. The added mass in this figure is normalized with the added mass of a single cylinder in unconfined flow $\left(=\rho \pi D^{2} L / 4\right)$.

The diagonal elements, which represent the added mass of cylinder i due to its own acceleration, are all above one. This is a consequence of the confinement effect. As the fluid is squeezed through gaps, the transverse flow velocities are higher than in the unconfined case. Consequently, the force required to accelerate the fluid is higher. The added mass in the direction orthogonal to the direction of the imposed acceleration is zero due to the symmetry of 


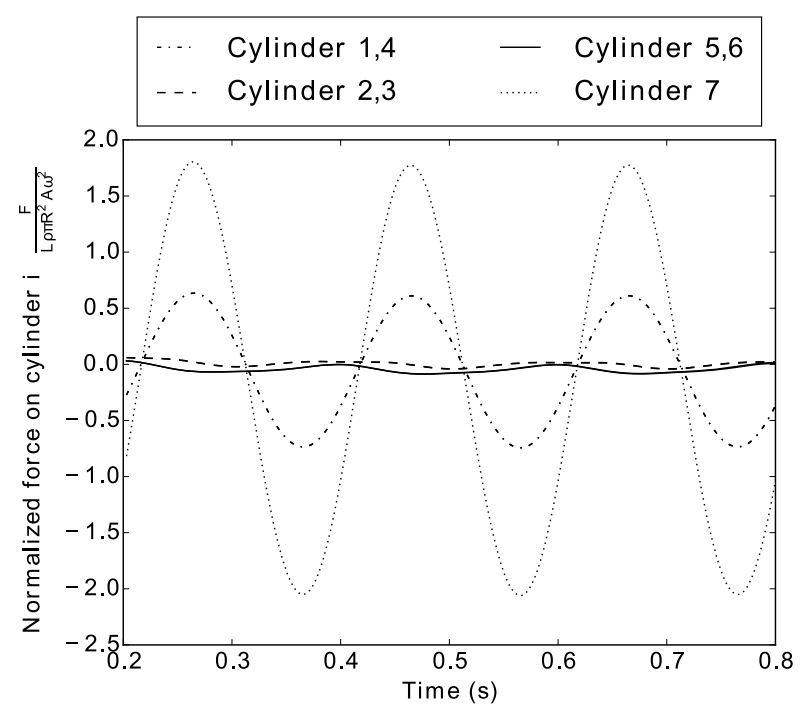

(a) URANS-results

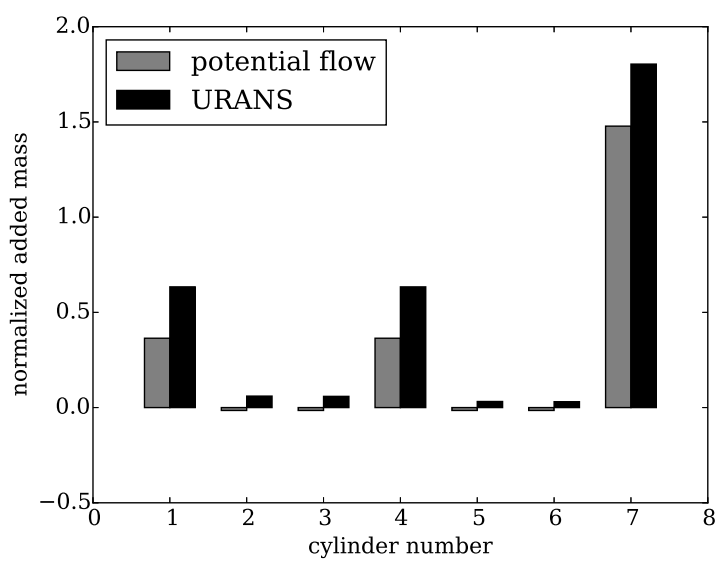

(b) Added mass comparison

Figure 6: Normalized force in the x-direction on all cylinders if the central cylinder (cylinder 7) undergoes a forced oscillation in the x-direction.

the geometry. Note that the added mass matrix itself is symmetric. If an acceleration of cylinder 1 imposes an inertia on cylinder $i$, it experiences the same inertia upon acceleration of cylinder $i$.

Figure 6a displays the normalized forces exerted on the different cylinders, resulting from an imposed sinusoidal motion of the central cylinder and computed by URANS. In contrast to potential flow, viscous and turbulence effects are also taken into account. Due to the high geometrical symmetry, the forces on cylinders 1-4, 2-3 and 5-6 are identical. From this graph, the inertial effect can be extracted as the component that is in phase with the imposed acceleration.

The comparison of the induced inertia computed by URANS or by potential flow theory is given in Figure 6b. It shows that both are predicting similar trends. However, the URANS simulations predict a significantly (20\%-40\%) higher force. This can be attributed to two effects. The first one is that, in order to have a convenient boundary condition in the potential flow theory, a circular external confinement is imposed, while in the URANS simulations a more realistic hexagonal confinement is used. However, the radius of the external circular confinement (12.95mm) is smaller than the average radius of the hexagonal confinement $(12.4 \mathrm{~mm}-14.3 \mathrm{~mm})$. The second effect is that turbulence mixing and viscous effects are dragging more fluid along. The turbulence mixing effect are modeled in the URANS simulation while they are not present in potential flow analysis. Consequently, the amount of added or hydrodynamic mass is higher in URANS simulations.

\subsubsection{Viscous forces}

The viscous force contribution in the linear theory is modeled with the empirical formulation in Equation 4. The local flow angle follows from the combination of a cylinder's own motion and the induced velocity by the presence and motion of neighboring cylinders. The induced velocity might be computed from potential flow theory and is given in Figure 7. The diagonal elements in this matrix represent the induced flow velocity by the cylinder's own motion. In contrast to an unconfined cylinder this is not zero, which is a consequence of the confinement induced by neighboring structures.

In the forced motion URANS-simulations, the viscous force corresponds to the part proportional to the imposed velocity. Note that this inertia - drag division is similar to the Morison decomposition (Morison et al., 1950). However, this does not imply that there are no viscous contributions to the inertia, as shown in the previous section.

Figure 8a displays the normal force proportional to the imposed velocity. The $C_{N}$-coefficient in the empirical data is fitted to the computed URANS result such that the normal force of the central cylinder equals the force from URANS-simulations. The resulting coefficient is 0.101 , which is similar to typical values for solitary cylinders. In a 


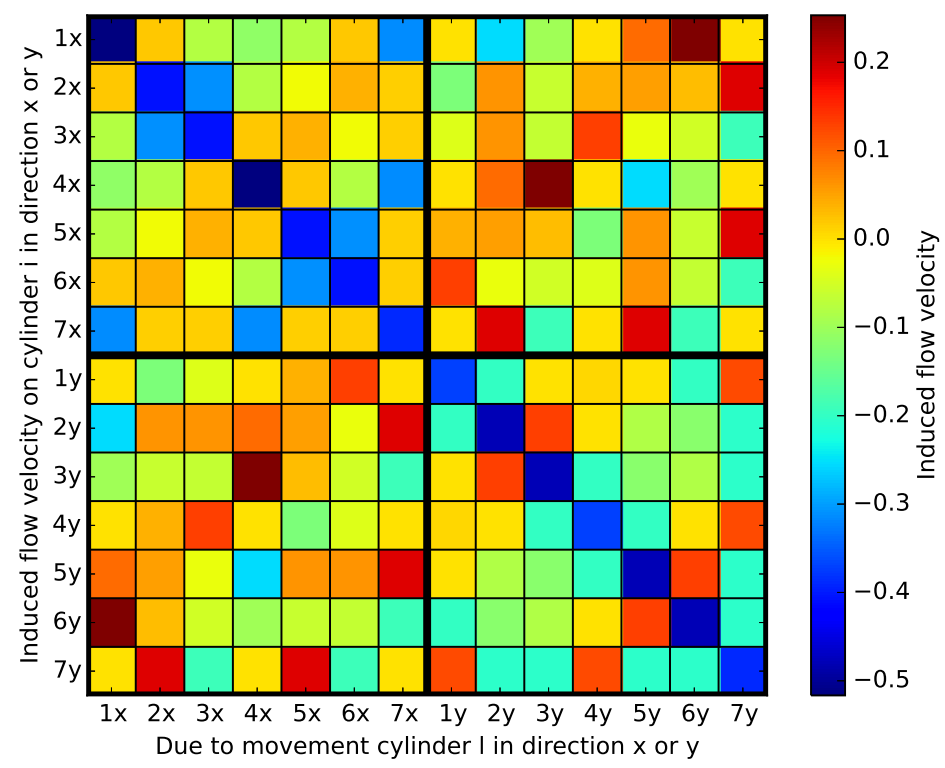

Figure 7: Induced velocity field, as predicted by potential flow theory.

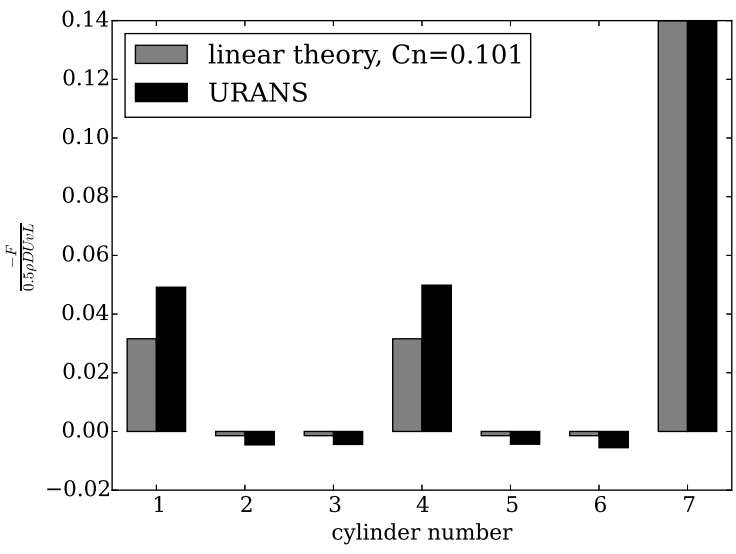

(a) The normal force, in phase with velocity, in the $\mathrm{x}$-direction upon oscillation of the central cylinder in the $\mathrm{x}$-direction.

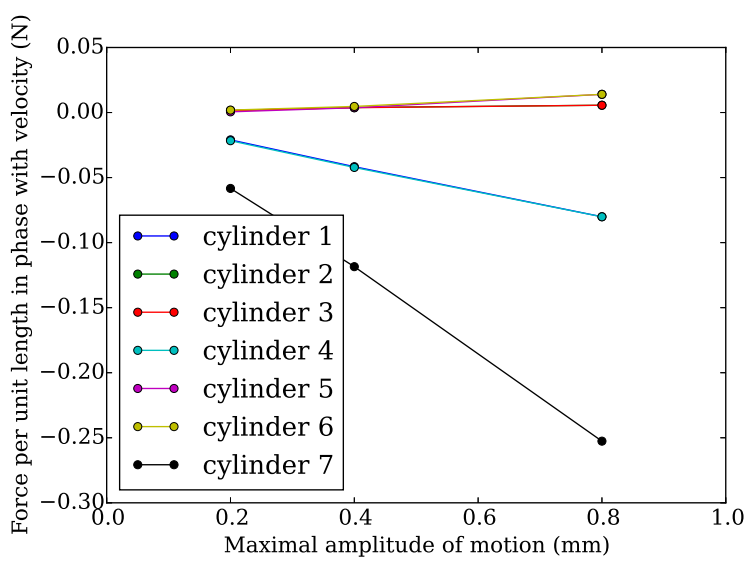

(b) Linear behavior of the normal force, in phase with velocity, as function of the maximal oscillation amplitude (the equivalent fraction of cross flow ranges from 0.003 to 0.013 ).

Figure 8: The linear region of the normal force in phase with velocity, computed by URANS and by linear theory. 


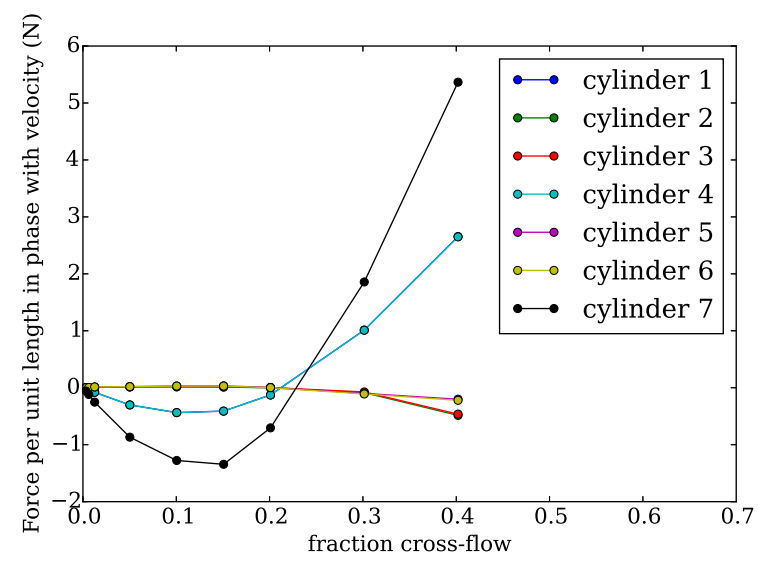

(a) The normal force, in phase with velocity, in the $\mathrm{x}$-direction upon oscillation of the central cylinder in the x-direction.

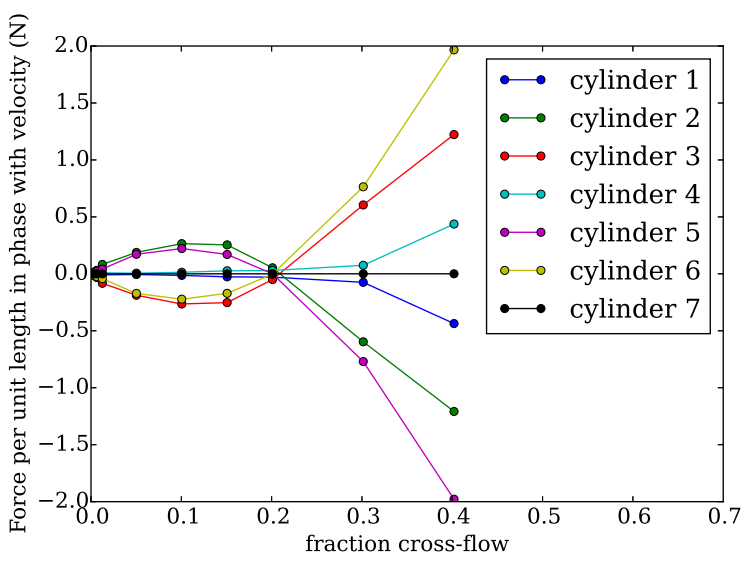

(b) The normal force, in phase with velocity, in the y-direction upon oscillation of the central cylinder in the x-direction.

Figure 9: The non-linear behavior of the drag force due to the forced oscillation of cylinder 7. The fraction cross-flow on the $\mathrm{x}$-axis is defined as the maximal velocity of cylinder 7 divided by the bulk axial velocity.

previous study (De Ridder et al., 2015) it was shown that this coefficient for a solitary cylinder ranges from 0.02 to 0.2 depending on the turbulence in the bulk flow. Divaret et al. (2014) measured a value of 0.11 and Ersdal and Faltinsen (2006) a value of 0.068 .

Although the fitted coefficient falls in the typical range, the viscous force predicted in linear theory on the neighboring cylinders is significantly lower than the URANS-predictions. As in the previous paragraph, this can be attributed to the two main differences between the two formulations. The first difference is the exterior confinement (hexagonal versus circular). The second difference is that due to turbulent mixing and viscosity effects, the velocity perturbations spread out further in the domain. This effect is modeled in URANS, while it is absent in the empirical formulation.

An important aspect of the empirical description is the linear behavior of the viscous force with respect to the apparent angle of attack. This behavior is verified with URANS simulations by varying the oscillation amplitude from $0.2 \mathrm{~mm}$ to $0.8 \mathrm{~mm}$. Combined with an oscillation frequency of $5 \mathrm{~Hz}$, which is close to the expected first mode frequency, the maximal transversal velocity ranges from $0.00628 \mathrm{~m} / \mathrm{s}$ to $0.0251 \mathrm{~m} / \mathrm{s}$ and the angle of attack is below two degrees. In this range, Figure $8 \mathrm{~b}$ shows that the viscous force indeed behaves linearly with apparent angle of attack on all cylinders.

\subsection{Non-linear viscous forces}

Solitary cylinders display this linear dependency of the viscous force on the angle of attack only if the apparent angle of attack is below 2 degrees (De Ridder et al., 2015; Divaret et al., 2014). At higher angles, the force becomes quadratically dependent on the angle of attack. Simultaneously, two counter-rotating vortices appear at the leeward side of the cylinder. In order to investigate if this transition also occurs on cylinders in arrays, the analysis from the previous section is extended to higher angles of attack.

As the distance between the different cylinders is only $1.8 \mathrm{~mm}$, the maximal velocity is increased by tuning the oscillation frequency while maintaining the amplitude at $0.2 \mathrm{~mm}$. The force in phase with velocity is shown in Figure 9. The $\mathrm{x}$-axis contains the fraction of cross-flow, which is defined as the maximal velocity of the central cylinder, divided by the bulk axial velocity. It is only a global measure for the amount of cross flow, as the local fraction of cross-flow differs significantly from this measure.

At low cross-flow fractions, Figure 9a shows that the force depends linearly on the transverse velocity. However, at fractions higher than 0.1 , a clear quadratic trend is visible. In contrast to the flow over a solitary cylinder, the quadratic part of the force on the central cylinder has the same sign as its velocity. Consequently, a destabilizing feedback is obtained at high cross-flow fractions, instead of having a damping effect. 

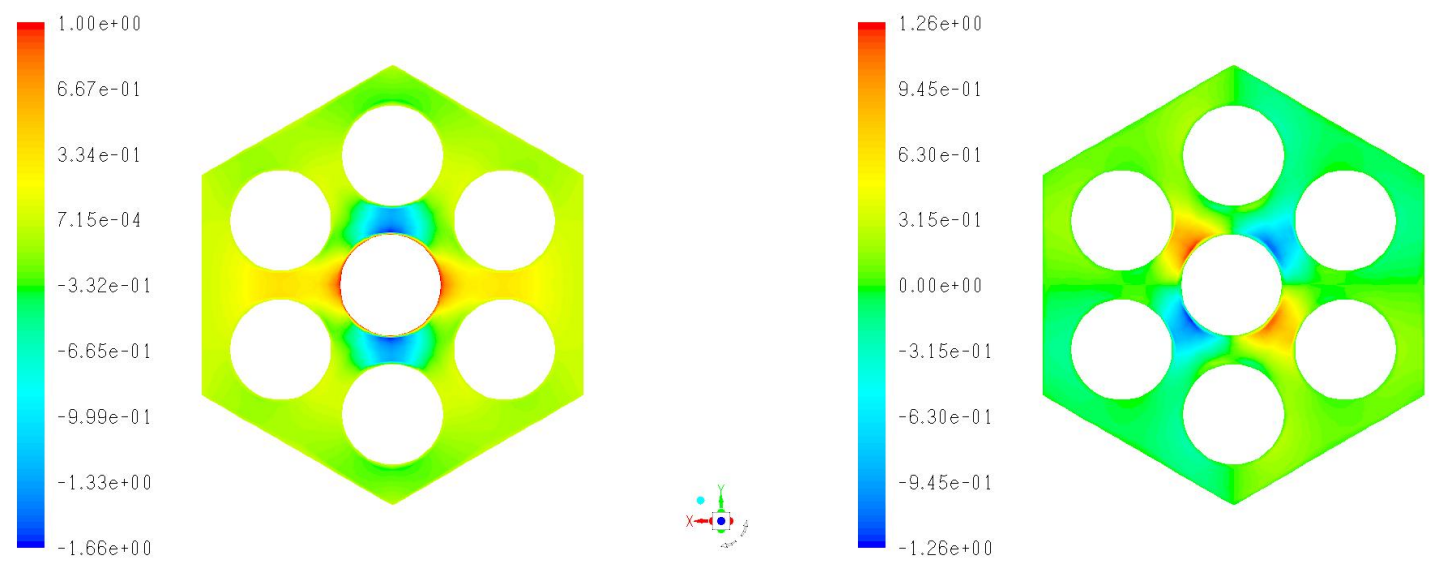

(a) Contours of $x$-velocity divided by the cylinder's speed.

(b) Contours of y-velocity divided by the cylinder's speed .
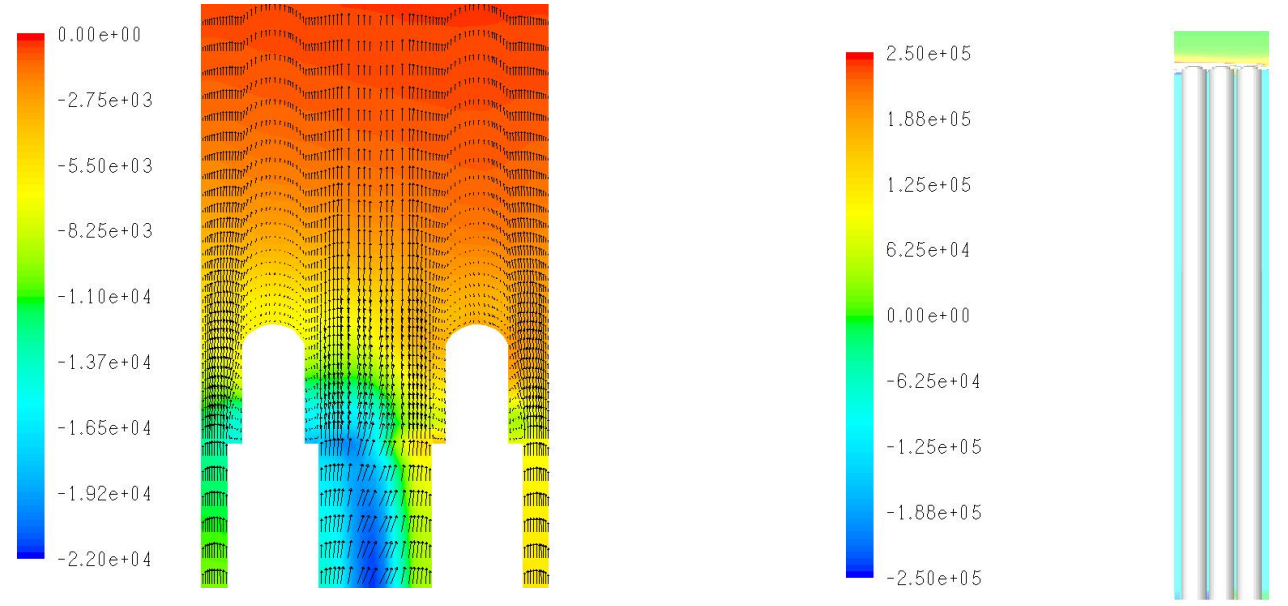

(c) Pressure contours $(\mathrm{Pa})$ close to the outlet of the bundle along with velocity vectors.

(d) Axial pressure gradient $(\mathrm{Pa} / \mathrm{m})$ through the bundle.
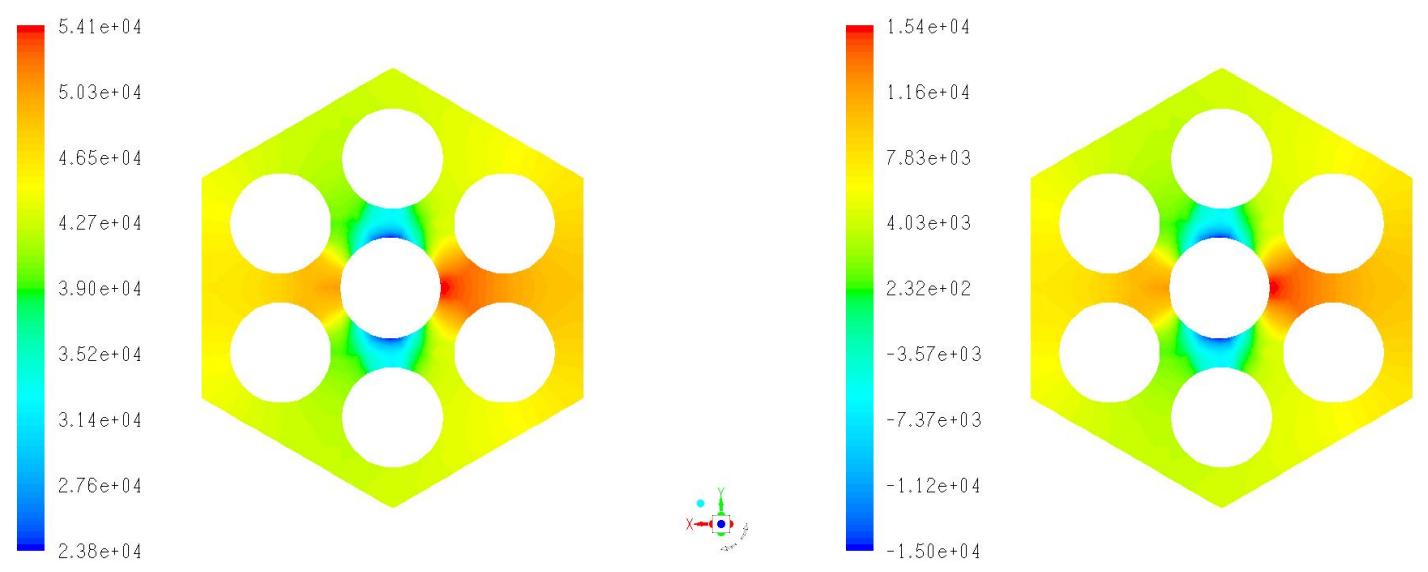

(f) Pressure contours in a cross-section at $\mathrm{z}=1.2 \mathrm{~m}$.

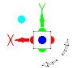


The $\mathrm{x}$ - and $\mathrm{y}$-velocity contours at $\mathrm{t}=1 / 4 \mathrm{~T}_{\mathrm{osc}}$ of the simulation with the highest transverse cylinder velocity are given in Figure 10a and 10b. Even at a cross-flow fraction of 0.4 or, equivalently, an angle of attack of $22^{\circ}$, the flow perturbation remains attached to the cylinder, as it would for low angles of attack. Note that around the central cylinder a net negative flow velocity (in the direction of the cylinder's momvement) is present, which is also predicted by potential flow theory.

The explanation for the quadratic force behavior can be found in the flow conditions in the outlet region. Figure $10 \mathrm{c}$ displays velocity vectors and pressure contours in a plane at $\mathrm{y}=4 \mathrm{~mm}$. It is observed from Figure 10a that if the central cylinder is moving to the left, the fluid has to go to the right in order to cross the cylinder. At the outlet of the bundle, the velocity field is seen to turn to a straight outlet flow. To enable this turning a pressure gradient in the $\mathrm{x}$-direction is present, as shown in Figure 10c. Consequently, the pressure at the right side of the figure is higher than at the left side. The required turning force increases quadratically with the transverse velocity component. Figure $10 \mathrm{~d}$ shows that in the bundle the axial pressure gradient is almost constant. The pressure difference is hence present throughout the entire bundle.

The pressure contours at different axial locations, which are displayed in Figures 10e and 10f, are consequently nearly identical. They only differ with a constant value, which is imposed by the pressure drop in axial direction through the bundle. At the left and right side of the central cylinder a high pressure area is present, which corresponds to the stagnation pressure. At the top and bottom a lower pressure area is present, as the velocity reaches its maximum in those locations. Note that the stagnation pressures at the left and right are modified by the earlier described pressure gradient, which is needed to turn the flow at the outlet.

The quadratic viscous forces with a positive feedback are present in URANS. They are not included in the linear model, which is hence only applicable to small amplitude and frequency motions. At higher amplitude and frequency motions, this quadratic force should be incorporated as well.

\section{Dynamics of a 7-rod bundle}

In this section the modal characteristics of the first and second mode group are reported. The results of the coupled CFD-CSM simulations are compared to linear theory.

\subsection{First mode group}

The dynamics of the first bending mode group in the stable region are shown in Figure 12. The first mode group refers to those modes that have the same first bending mode deflection along the axial direction. Although all the modes in a group have per definition the same bending shape, every mode combines motions in a different pattern when looking at a cross-section through the bundle. An example of these patterns is given in Figure 11 . This means that a 7-cylinder cluster can have up to 14 modes per group.

In the numerical simulations, 9 initial perturbations have been used per mode group. Each perturbation corresponds to an eigenmode predicted by linear theory (without damping). The simulated frequencies as function of the bulk flow velocity are shown in Figure 12a. Due to the symmetry, only 9 modes are present instead of 14 . At a flow velocity of $1 \mathrm{~m} / \mathrm{s}$, the simulations also predict a range of eigenfrequencies: from $22 \mathrm{rad} / \mathrm{s}$ to $37 \mathrm{rad} / \mathrm{s}$. This range is a consequence of the added inertia, which was discussed in detail in Section 3.2.1. Note that the eigenfrequency of a solitary cylinder subjected to a similar confinement falls in this range.

The eigenfrequency range is also predicted by linear theory (with damping), as shown in Figure 12b. Compared to the simulation results in Figure 12a, the predicted range is smaller. This observation is in line with the results in Section 3.2.1, which show that the k- $\omega$ SST URANS-simulations predicted a higher added mass than potential flow theory, both on the cylinder being accelerated and on neighboring cylinders.

At increasing flow velocity, three trends are visible on the frequency graphs. Firstly, the eigenfrequency of the highest frequency modes are increasing with flow velocity, while, secondly, the eigenfrequency of the lowest frequency modes are decreasing with flow velocity. Finally, linear theory shows that at higher flow velocity all 14 modes become visible. This is a consequence of the symmetry breaking of the damping terms. At low flow velocities, duplicate eigenfrequencies are present.

The decrease of the eigenfrequency of the lowest frequency modes is a result of the inviscid forces in Equation 2. The centrifugal force component in this equation is proportional to the second spatial derivative of the deflection 

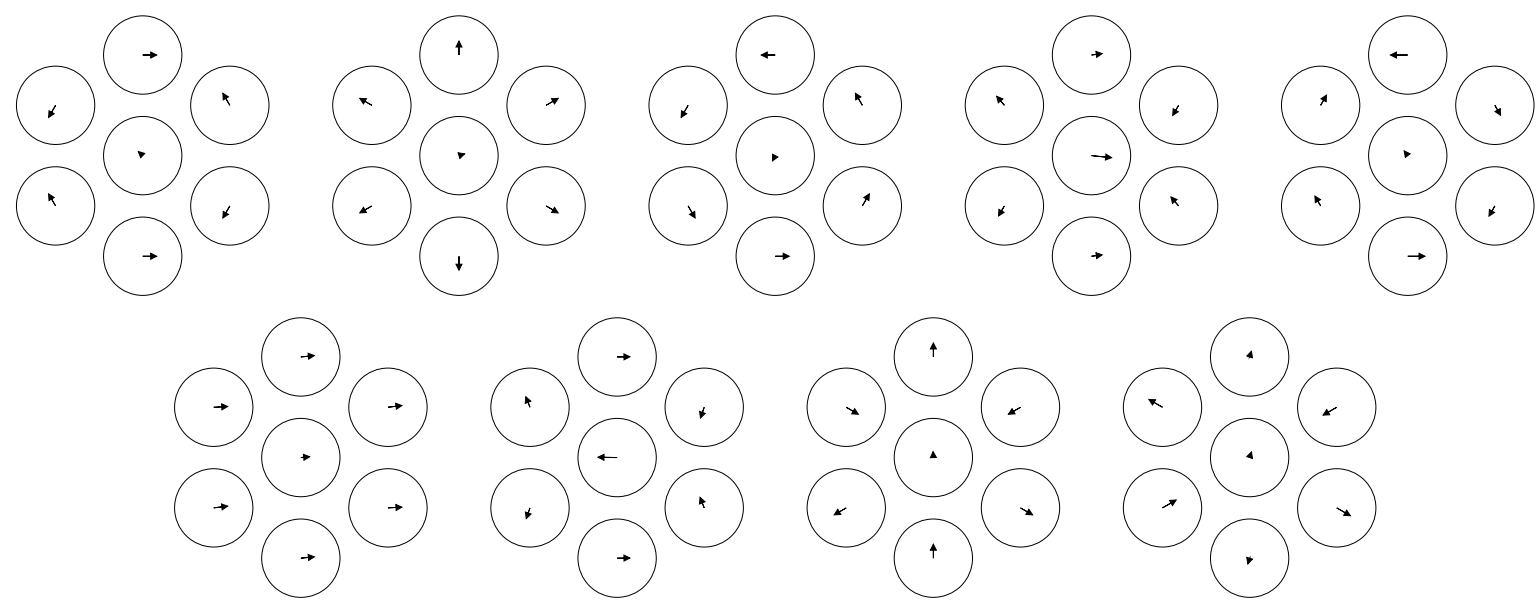

Figure 11: Cross-sectional view of the nine initial perturbations. Every perturbation corresponds to a mode obtained from linear theory. A mode group consists of all those modes which have the same radial deflection along the axial direction, but with different cross-sectional patterns.

and on the axial flow velocity squared. Furthermore, it is opposing the stiffness contribution. Consequently, at higher flow velocities a lower apparent stiffness is visible. At even higher flow velocity (not shown on the graph), the eigenfrequency goes to zero, and the cylinders will buckle. Note that a solitary cylinder behaves similarly (De Ridder et al., 2015; Modarres-Sadeghi et al., 2008).

However, the highest frequency modes follow the opposite trend. The increase in eigenfrequency is a result of tension increase due to the wall shear stress of the fluid flow. In linear theory this is modeled with a term proportional to $0.5 \rho_{f} u^{2} c_{f} D(L-x) \frac{\partial u}{\partial x}$, with the $c_{f}$-coefficient tuned to the URANS-simulations. This shows that an increase in flow velocity can increase or decrease the apparent stiffness, depending on whether the shear stress or the centrifugal force is largest. The higher frequency modes in Figures $12 \mathrm{a}$ and $12 \mathrm{~b}$ experience less added mass than lower frequency modes. Equation 2 shows that the centrifugal fluid force behaves similar to the added mass. Consequently, less centrifugal force is acting on higher frequency modes. This explains why some modes show an increase in eigenfrequency and others a decrease. This also implies that the buckling instability will only appear in a few modes (those with the lowest eigenfrequencies).

Figure $12 \mathrm{c}$ and $12 \mathrm{~d}$ display the damping measured from the simulations and the damping predicted by linear theory as a function of flow velocity. Similarly to the eigenfrequency, the linear theory predicts a narrower band of damping values. This is similar to the fluid force results in Section 3.2.2, in which it was shown that the coupling component of the normal viscous force is smaller in linear theory. There are two reasons for this difference: the transverse (in $\mathrm{x}$ - or $\mathrm{y}$-direction) velocity disturbance in potential flow theory is not affected by turbulent mixing, while this effect is modeled in URANS-simulations. The second reason is that the axial flow velocity is not uniform in a cross-section through the bundle, as shown in Figure 4a. Note that this non-uniformity also affects centrifugal forces. Despite these differences between linear theory and simulations, both predict an almost linear increase of damping with flow velocity. This implies that the viscous forces are in the linear regime.

\subsection{Higher modes}

Figure 13 shows the modal characteristics of both the first and second mode group as a function of axial bulk flow velocity. As in the previous paragraph for the first mode frequencies, a range of second eigenmode frequencies is present. At a flow speed of $1 \mathrm{~m} / \mathrm{s}$, the ratio of maximum frequency over minimum frequency of the first mode group is 1.73 , which is almost identical to the ratio of the second mode group (1.74).

Compared to the simulation results, the linear theory predicts a smaller range of eigenfrequencies, which is a consequence of the smaller amount of cross-coupling of inertial effects, as discussed in the previous paragraph. The damping of the second mode group increases linearly with flow velocity, both in coupled CFD-CSM simulations as well as in linear theory. The simulations predict a larger difference between the first and second group damping than linear theory. The reason for this difference might be that in reality the normal force coefficient is not entirely constant 


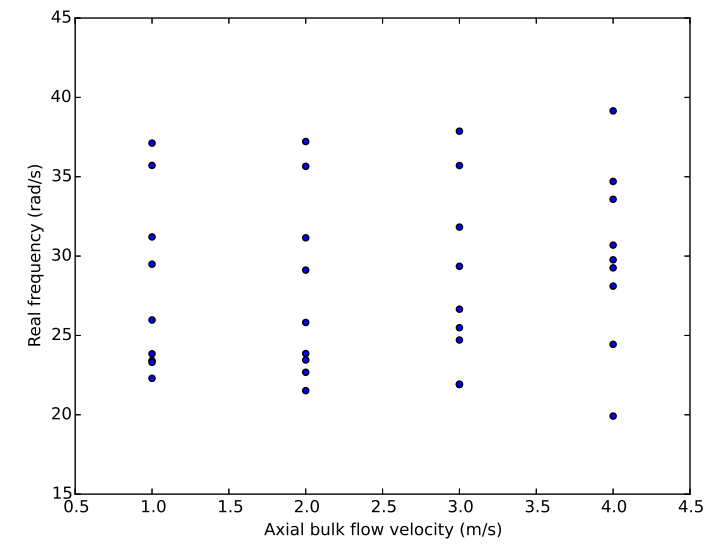

(a) First mode frequency (rad/s) computed with coupled simulations.

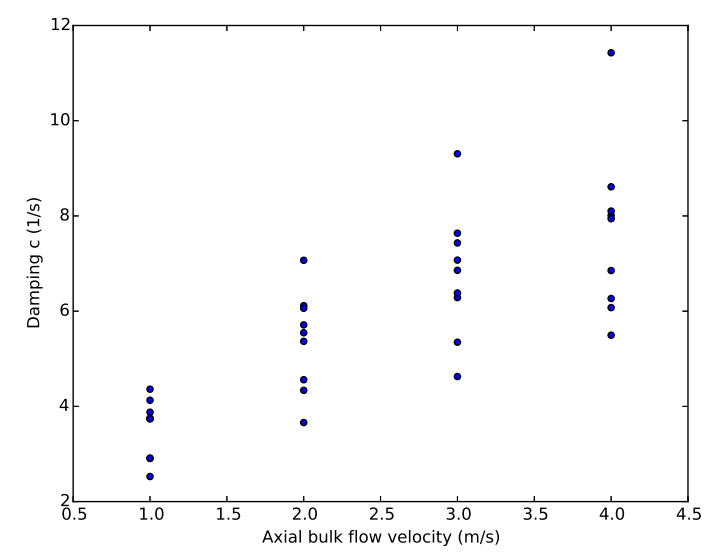

(c) First mode damping (1/s) computed with coupled simulations.

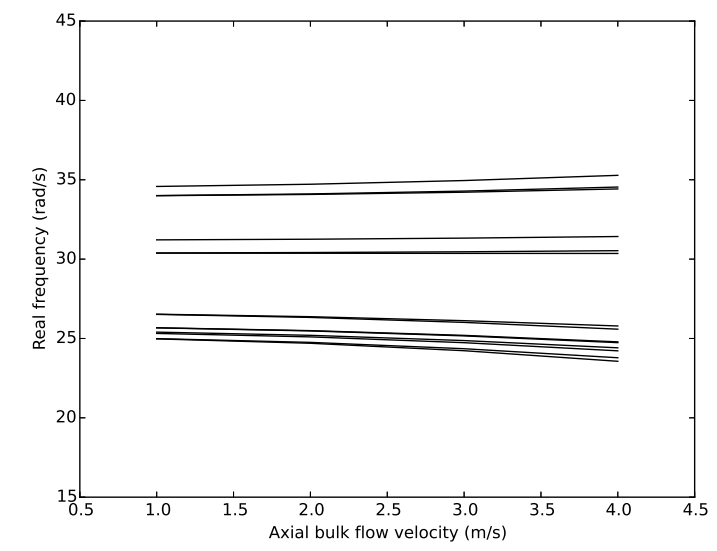

(b) First mode frequency (rad/s) based on linear theory.

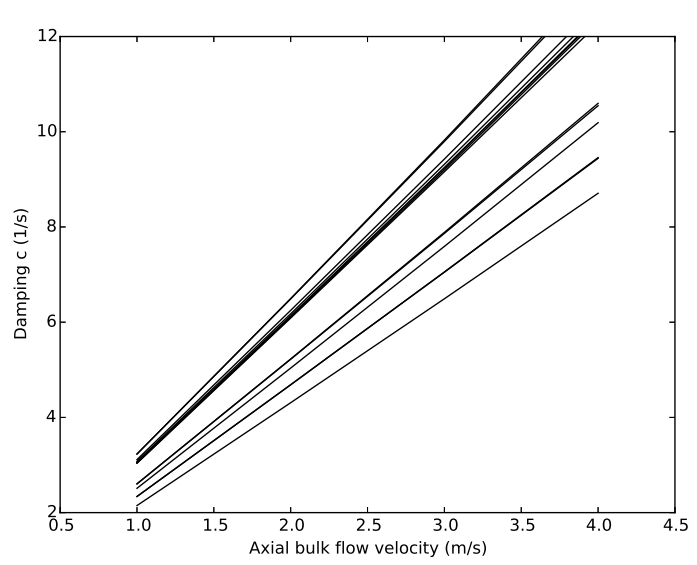

(d) First mode damping (1/s) based on linear theory.

Figure 12: Modal characteristics of the first mode group as function of the axial bulk flow velocity. 


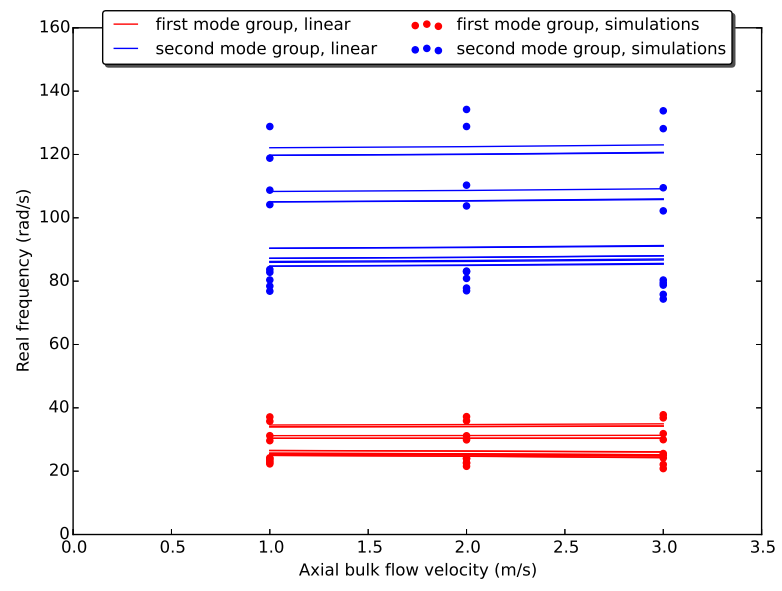

(a) Frequency.

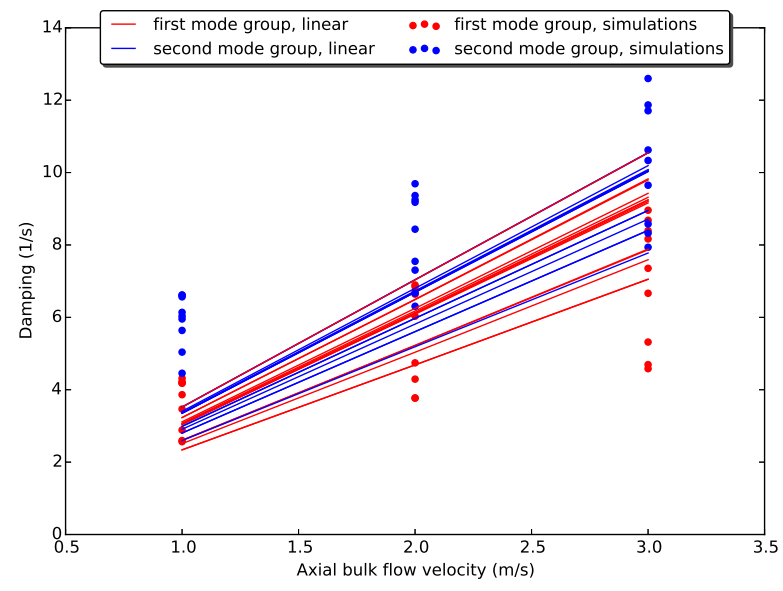

(b) Damping.

Figure 13: Comparison between modal characteristics of the first and second mode group as function of axial bulk flow velocity.

along the length of the cylinder. Consequently, the different mode groups experience slightly different apparent (or averaged) force coefficients.

\section{One cylinder approximations}

The major drawback of the simulations in the previous paragraph is the scaling of computational time with the number of cylinders in the bundle. Generally, the number of modes within a group scales linearly with the number of cylinders in the bundle. That means that the computational cost scales quadratically with the number of cylinders as both the number of modes and the domain size increase linearly.

Therefore, it is useful to investigate reduced models. A first approach is to use a reduced physics model, such as the linear theory described in Section 2.1. The drawback is that empirical coefficients are required and that the effect of mixing on the hydrodynamic coupling is unknown. The coefficients can eventually be found from numerical simulations, as done in Section 3.2.2. The potential flow solution also scales quadratically with the number of cylinders, but it is per cylinder (orders of magnitude) faster than the URANS-based simulations.

A second approach is to find bounding limits to the resonance frequencies and damping ratio's, which might be sufficient in practical applications. In a previous paper (De Ridder et al., 2013), it was hypothesized that those limits could be found by using a periodic building block unit as fluid domain. By imposing either periodic or zero velocity gradient conditions on the sides of the hexagonal building block, either the upper or lower limit can be found. The reasoning is that periodic conditions mimic those modes that have little added mass. It was shown that the flow velocity perturbation due the cylinder's movement is smaller or equal to the cylinder's velocity, with a relatively small region being affected by it. By imposing symmetric (zero velocity gradient) conditions, the flow perturbation is higher than the cylinder's velocity and in the opposite direction. It also affects the entire fluid area, giving rise to a very large confinement effect. Although, the symmetry boundary conditions are not necessarily representing physically possible modes, they impose the largest confinement possible and consequently the lowest possible eigenfrequencies.

The modal characteristics of the first mode group obtained using a smaller fluid domain with either periodic or symmetric conditions are given in Figure 14. The characteristics obtained by explicit simulations of 7 cylinders are represented by a bounding trapezium. Figure 14a indeed shows that the single cylinder simulations provide reasonable upper and lower limits for the resonance frequency. Additionally, the single cylinder simulations also give upper and lower bounds estimations for the damping, as shown in Figure 14b, as expected earlier (De Ridder et al., 2013). The drawback of one cylinder simulations is that the shear stress on a cylinder is different from the shear stress in a bundle. This explains the decreasing natural frequency trend of the simulation with periodic boundary conditions instead of an increase as expected from the 7-rod bundle simulation. The difference in hydraulic diameter also causes 


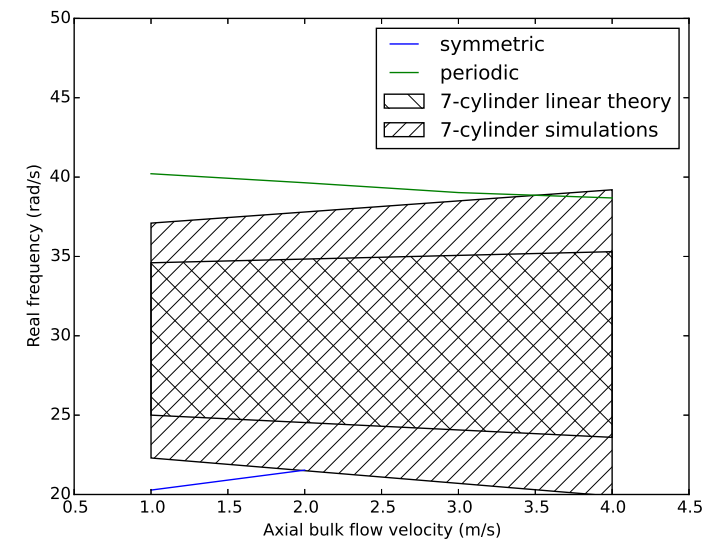

(a) Eigenfrequency of first mode group.

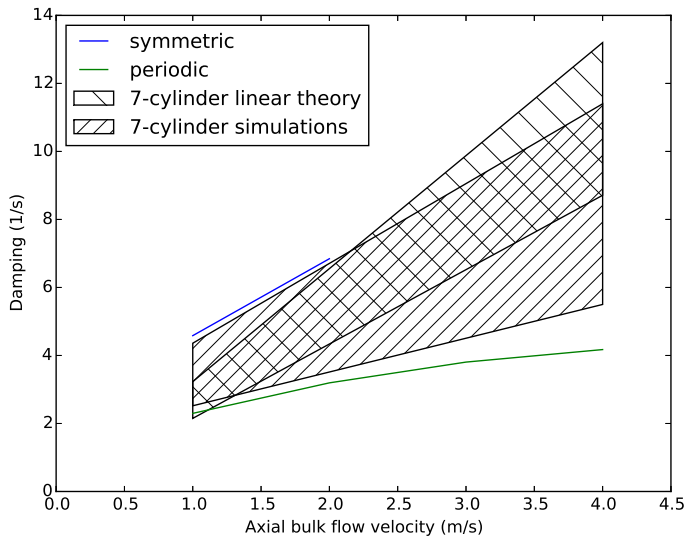

(b) First mode damping of first mode group.

Figure 14: Bounds of modal characteristics of the first mode group as function of axial bulk flow velocity. At axial velocities above $2 \mathrm{~m} / \mathrm{s}$, the free vibration decay of the cylinder, enclosed in a fluid domain with symmetric boundary conditions, starts developping a flutter instability (cf. Figure $15)$.

the simulations with symmetric boundary conditions to no longer predict an accurate lower bound at velocities above $2 \mathrm{~m} / \mathrm{s}$.

The centerline displacement for symmetric boundary conditions at higher flow velocities are shown in Figure 15. It shows that at $\mathrm{u}=4 \mathrm{~m} / \mathrm{s}$, a flutter instability occurs, while the simulations with 7 rods were always in the stable region. This indicates that the simulation with symmetric side conditions might be too confining and hence it predicts a fluidelastic instability at too low flow velocities. The one cylinder simulation are thus useful approximations for the characteristics of a cylinders in a bundle, although the difference in hydraulic diameter might limit the accuracy.

In conclusion, the one cylinder approximations provide useful estimations for the upper and lower limits of resonance frequency and damping, although some error is to be expected when comparing to characteristics of an entire bundle.

\section{Conclusions}

The comparison between inertial forces coming from URANS simulations and from potential flow analysis showed that both methods predict qualitatively the same behavior, although the coupling effect is weaker in potential flow analysis. The higher inertia in URANS simulations is attributed to the mixing effect of turbulence, which spread flow perturbations due to accelerations of cylinders to a larger fraction of the domain.

Because the perturbations extend further in URANS simulations than in potential flow analysis, the normal/damping forces are also underestimated in linear theory. Additionally, it has been shown that a non-linear destabilizing contribution is present in the forces proportional to the cylinder's transverse velocity.

In the second part, FSI-simulations of a bundle of flexible cylinders are performed. FSI-simulations of free vibration decays of mode shapes following from linear theory allow the estimation of modal characteristics. In contrast to linear theory, these simulations take into account differences in axial flow velocity between subchannels and gaps, mixing effects and non-linear effects (if any occur). Despite quantitative differences, linear theory predicts similar trends in modal characteristics as coupled FSI-simulations, provided that appropriate coefficients are used. In this case the damping coefficient in linear theory was tuned to the simulations to have good agreement. The important difference between linear theory and FSI-simulations is that the predicted range of eigenfrequencies and damping is smaller in linear theory.

The modal characteristics followed similar trends with increasing flow velocity as a solitary cylinder. The damping increases almost linearly with flow velocity. The eigenfrequency of the modes could either increase or decrease with flow velocity, depending on the relative magnitude of centrifugal force compared to the tensioning effect of friction. 

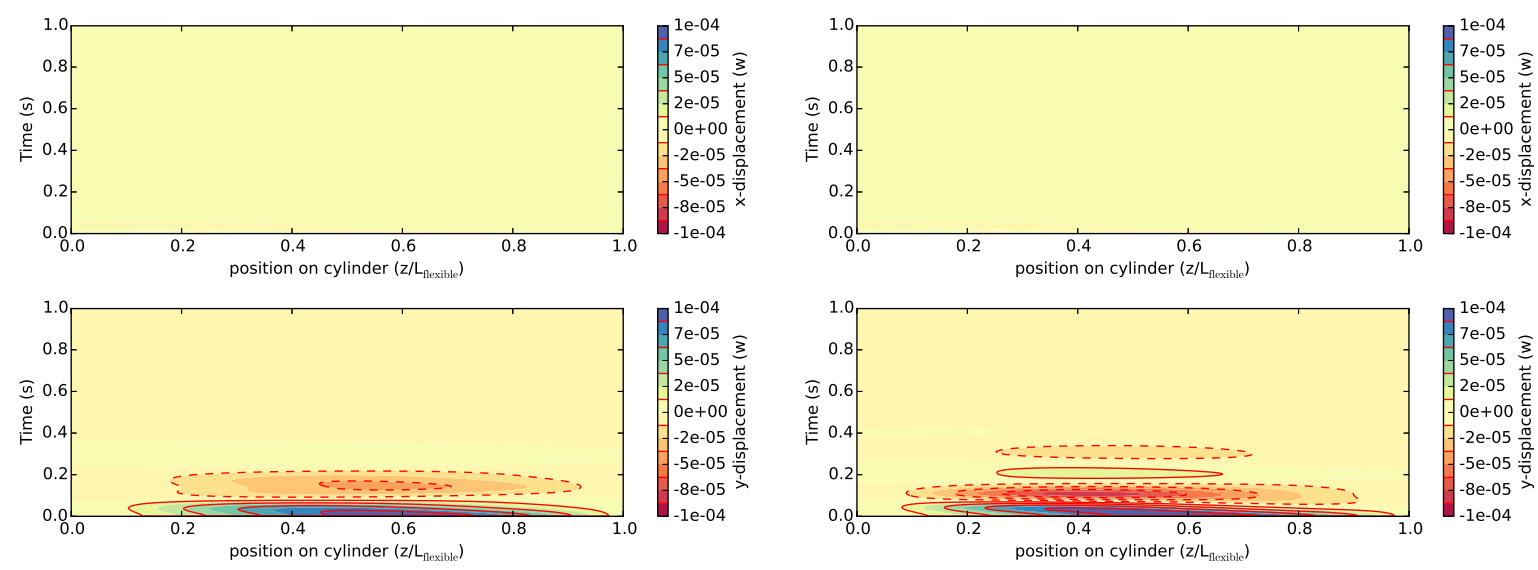

(a) $\mathrm{u}=2 \mathrm{~m} / \mathrm{s}$.

(b) $\mathrm{u}=3 \mathrm{~m} / \mathrm{s}$.
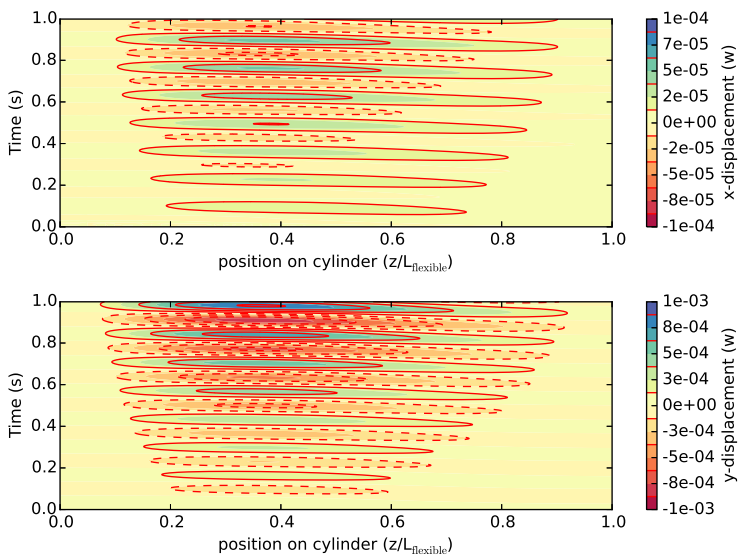

(c) $\mathrm{u}=4 \mathrm{~m} / \mathrm{s}$.

Figure 15: Free vibration decay of 1st mode perturbation as a function of bulk axial flow velocity (all displacements in m). At low flow velocity a fast decay is observed, while at high flow velocities, a flutter instability can be observed. 
As simulations of cylinder arrays become more expensive as the number of cylinders increases, simulations which provide bounding limits to the resonance spectra were performed. It was shown that by imposing periodic flow boundary conditions on the side of a periodic unit cell with one cylinder, the maximal resonance frequency and minimum damping was obtained. By imposing that the flow perturbations remains inside the periodic unit cell, the minimal resonance frequency (highest added mass) and maximal damping was obtained. The drawback of one cylinder simulations is that the shear stress is different from the shear stress in a bundle, as the hydraulic diameter is different.

\section{Acknowledgments}

This work was performed in the framework of the Horizon 2020 MYRTE Project. It has received funding from the Euratom research and training program 2014-2018 under grant agreement No 662186 (MYRTE).

\section{Appendix A. Hydrodynamic inviscid coupling by a potential flow analysis}

This section is based on the derivation by Païdoussis and Suss 1977 and Païdoussis 1977. Upon assuming that the displacements of the cylinders are small, it is reasonable to assume that the cylinders stay parallel to each other. In that case, the problem reduces to determining the hydrodynamic field in a two-dimensional section. The second assumption is to use potential flow theory and hence ignore all viscous and rotational flow effects.

Using this approach, any fluid velocity vector can be written as $\vec{v}=\nabla \phi$, where the velocity potential satisfies

$$
\nabla^{2} \phi=0,
$$

with the boundary conditions requiring that (a) the velocity normal to the surface of each cylinder equals the velocity of the cylinder in that direction and (b) the velocity normal to the exterior flow channel is zero on its surface.

The total potential $\phi$ can be written as a sum of potentials $\phi_{j}$, due to the presence and/or motion of cylinder $j$ in the channel, resulting in

$$
\phi=\sum_{j=1}^{K} \phi_{j}
$$

with $K$ the number of cylinders in the flow channel. Due to the linearity of Equation A.1, the contributing potentials satisfy

$$
\nabla^{2} \phi_{j}=0
$$

The solution of this equation has the following form

$$
\phi_{j}\left(r_{j}, \theta_{j}\right)=\sum_{n=1}^{\infty} A_{n j} r_{j}^{n} \cos \left(n \theta_{j}\right)+B_{n j} r_{j}^{n} \sin \left(n \theta_{j}\right)+C_{n j} r_{j}^{-n} \cos \left(n \theta_{j}\right)+D_{n j} r_{j}^{-n} \sin \left(n \theta_{j}\right),
$$

with $r_{j}$ and $\theta_{j}$ cylindrical coordinates centered around cylinder $j$. In order to apply boundary conditions, the potential due to cylinder $j$ has to be written in terms of coordinates centered on cylinder $i$. This can be done by the following coordinate transform:

$$
r_{j} e^{\imath \theta_{j}}=r_{i} e^{\imath \theta_{i}}-P_{i j} e^{\imath \psi_{i j}},
$$

where $P_{i j}$ is the center-to-center distance of the cylinders and $\psi_{i j}$ the polar angle of cylinder $j$ in a coordinate system centered on cylinder $i$. Note that the real part of $\left(r_{j} e^{\imath \theta_{j}}\right)^{n}$ corresponds to $r_{j}^{n} \cos \left(n \theta_{j}\right)$, the imaginary $\operatorname{part} \operatorname{to} r_{j}^{n} \sin \left(n \theta_{j}\right)$ in Equation A.4 and similar for $\left(r_{j} e^{-\imath \theta_{j}}\right)^{-n}$. Note that Equation A.4 can be rewritten as

$$
\phi_{j}\left(r_{j}, \theta_{j}\right)=\sum_{n=1}^{\infty} A_{n j} \mathfrak{R}\left(r_{j} e^{\imath \theta_{j}}\right)^{n}+B_{n j} \mathfrak{J}\left(r_{j} e^{\imath \theta_{j}}\right)^{n}+C_{n j} \mathfrak{R}\left(r_{j} e^{-\imath \theta_{j}}\right)^{-n}+D_{n j} \mathfrak{J}\left(r_{j} e^{-\imath \theta_{j}}\right)^{-n} .
$$


By substituting Equation A.5 in the previous expression and applying the generalized binomial of Newton $\left((x+y)^{r}=\right.$ $\left.\sum_{m=0}^{r}\left(\begin{array}{c}r \\ m\end{array}\right) x^{r-m} y^{m}\right)$ if $|x|>|y|$, Equation A.6 becomes

$$
\begin{aligned}
\phi_{j}^{i}\left(r_{i}, \theta_{i}\right) & =\sum_{n=1}^{\infty} A_{n j} \mathfrak{R}\left(\sum_{m=0}^{n}\left(\begin{array}{l}
n \\
m
\end{array}\right)\left(r_{i} e^{\imath \theta_{i}}\right)^{m}\left(-P_{i j} e^{\imath \psi_{i j}}\right)^{n-m}\right)+B_{n j} \mathfrak{J}\left(\sum_{m=0}^{n}\left(\begin{array}{l}
n \\
m
\end{array}\right)\left(r_{i} e^{\imath \theta_{i}}\right)^{m}\left(-P_{i j} e^{\imath \psi_{i j}}\right)^{n-m}\right) \\
+ & C_{n j} \mathfrak{R}\left(\sum_{m=0}^{\infty}\left(\begin{array}{c}
-n \\
m
\end{array}\right)\left(r_{i} e^{-\imath \theta_{i}}\right)^{m}\left(-P_{i j} e^{-\imath \psi_{i j}}\right)^{-n-m}\right)+D_{n j} \mathfrak{J}\left(\sum_{m=0}^{\infty}\left(\begin{array}{c}
-n \\
m
\end{array}\right)\left(r_{i} e^{-\imath \theta_{i}}\right)^{m}\left(-P_{i j} e^{-\imath \psi_{i j}}\right)^{-n-m}\right) .
\end{aligned}
$$

Note that the first two terms follow directly from the standard binomial. Equation A.7 is valid if $r_{i}<P_{i j}$. If $r_{i}$ exceeds $P_{i j}$, the following expression is valid:

$$
\begin{array}{r}
\phi_{j}^{i}\left(r_{i}, \theta_{i}\right)=\sum_{n=1}^{\infty} A_{n j} \mathfrak{R}\left(\sum_{m=0}^{n}\left(\begin{array}{l}
n \\
m
\end{array}\right)\left(r_{i} e^{\imath \theta_{i}}\right)^{m}\left(-P_{i j} e^{\imath \psi_{i j}}\right)^{n-m}\right)+B_{n j} \mathfrak{J}\left(\sum_{m=0}^{n}\left(\begin{array}{l}
n \\
m
\end{array}\right)\left(r_{i} e^{\imath \theta_{i}}\right)^{m}\left(-P_{i j} e^{\imath \psi_{i j}}\right)^{n-m}\right) \\
+C_{n j} \mathfrak{R}\left(\sum_{m=0}^{\infty}\left(\begin{array}{c}
-n \\
m
\end{array}\right)\left(r_{i} e^{-\imath \theta_{i}}\right)^{-n-m}\left(-P_{i j} e^{-\imath \psi_{i j}}\right)^{m}\right)+D_{n j} \mathfrak{J}\left(\sum_{m=0}^{\infty}\left(\begin{array}{c}
-n \\
m
\end{array}\right)\left(r_{i} e^{-\imath \theta_{i}}\right)^{-n-m}\left(-P_{i j} e^{-l \psi_{i j}}\right)^{m}\right) .
\end{array}
$$

It is convenient to rewrite Equations A.7 and A.8 in trigonometric form:

$$
\begin{aligned}
\phi_{j}^{i}\left(r_{i}, \theta_{i}\right)= & \sum_{n=1}^{\infty} \sum_{m=0}^{n}\left(\begin{array}{l}
n \\
m
\end{array}\right) \frac{\left(r_{i}\right)^{m}}{\left(-P_{i j}\right)^{m-n}}\left(A_{n j} \cos \left(m \theta_{i}+(n-m) \psi_{i j}\right)+B_{n j} \sin \left(m \theta_{i}+(n-m) \psi_{i j}\right)\right) \\
& +\sum_{m=0}^{\infty}\left(\begin{array}{c}
-n \\
m
\end{array}\right) \frac{\left(r_{i}\right)^{m}}{\left(-P_{i j}\right)^{n+m}}\left(C_{n j} \cos \left(m \theta_{i}-(n+m) \psi_{i j}\right)-D_{n j} \sin \left(m \theta_{i}-(n+m) \psi_{i j}\right)\right)
\end{aligned}
$$

and

$$
\begin{aligned}
\phi_{j}^{i}\left(r_{i}, \theta_{i}\right) & =\sum_{n=1}^{\infty} \sum_{m=0}^{n}\left(\begin{array}{l}
n \\
m
\end{array}\right) \frac{\left(r_{i}\right)^{m}}{\left(-P_{i j}\right)^{m-n}}\left(A_{n j} \cos \left(m \theta_{i}+(n-m) \psi_{i j}\right)+B_{n j} \sin \left(m \theta_{i}+(n-m) \psi_{i j}\right)\right) \\
& +\sum_{m=0}^{\infty}\left(\begin{array}{c}
-n \\
m
\end{array}\right) \frac{\left(-P_{i j}\right)^{m}}{\left(r_{i}\right)^{n+m}}\left(C_{n j} \cos \left(-(n+m) \theta_{i}+m \psi_{i j}\right)-D_{n j} \sin \left(-(n+m) \theta_{i}+m \psi_{i j}\right)\right) .
\end{aligned}
$$

The total potential written in terms of the coordinate system of cylinder $i$ equals

$$
\phi^{i}\left(r_{i}, \theta_{i}\right)=\sum_{j=1}^{K *} \phi_{j}^{i}\left(r_{i}, \theta_{i}\right)+\phi_{i}\left(r_{i}, \theta_{i}\right)
$$

where $K$ is the number of cylinders and the $*$ indicates that the summation is taken over all elements except for element $i$.

\section{Appendix A.1. Boundary conditions on the cylinders in the channel}

As the fluid flow has to follow the motion of the cylinder, the radial derivative of the (total) potential on the surface of cylinder $i$ satisfies

$$
\left.\frac{\partial \phi^{i}}{\partial r_{i}}\right|_{r_{i}=R_{i}}=\frac{\partial u_{i}}{\partial t} \cos \left(\theta_{i}\right)+\frac{\partial v_{i}}{\partial t} \sin \left(\theta_{i}\right), \quad \text { for } i=1 . . K .
$$

In this equation, $u_{i}$ is the displacement of cylinder $i$ in $\mathrm{x}$-direction and $v_{i}$ in the y-direction. Similarly, no flow goes through the exterior confinement

$$
\left.\frac{\partial \phi_{j}^{0}}{\partial r_{0}}\right|_{r_{0}=R_{0}}=0, \text { for } j=1 . . K
$$


where the index 0 stands for the exterior cylindrical confinement. Upon substituting Equation A.9 in Equation A.11, the derivative of the total potential expressed in a coordinate system attached to cylinder $i$ becomes

$$
\begin{aligned}
\left.\frac{\partial \phi^{i}}{\partial r_{i}}\right|_{r_{i}=R_{i}}= & \sum_{j=1}^{K *} \sum_{n=1}^{\infty} \sum_{m=0}^{n}\left(\begin{array}{c}
n \\
m
\end{array}\right) \frac{m\left(R_{i}\right)^{m-1}}{\left(-P_{i j}\right)^{m-n}}\left(A_{n j} \cos \left(m \theta_{i}+(n-m) \psi_{i j}\right)+B_{n j} \sin \left(m \theta_{i}+(n-m) \psi_{i j}\right)\right)+ \\
& \sum_{j=1}^{K *} \sum_{n=1}^{\infty} \sum_{m=0}^{\infty}\left(\begin{array}{c}
-n \\
m
\end{array}\right) \frac{m\left(R_{i}\right)^{m-1}}{\left(-P_{i j}\right)^{n+m}}\left(C_{n j} \cos \left(m \theta_{i}-(n+m) \psi_{i j}\right)-D_{n j} \sin \left(m \theta_{i}-(n+m) \psi_{i j}\right)\right)+ \\
& \sum_{n=1}^{\infty} n R_{i}^{n-1}\left(A_{n i} \cos \left(n \theta_{i}\right)+B_{n i} \sin \left(n \theta_{i}\right)\right)-n R_{i}^{-n-1}\left(C_{n i} \cos \left(n \theta_{i}\right)+D_{n i} \sin \left(n \theta_{i}\right)\right)
\end{aligned}
$$

Note that the $m=0$ term disappears in the previous equation. In order to employ the independence of $\cos \left(m \theta_{i}\right)$ amongst each other and the independence of $\left(\cos \left(m \theta_{i}\right)\right.$ and $\left.\sin \left(m \theta_{i}\right)\right)$, Equation A.14 has to be rewritten in a form which easily separates those terms. Therefore, the following summation identity is used:

$$
\sum_{n=a}^{b} \sum_{m=a}^{n} x_{m, n}=\sum_{m=a}^{b} \sum_{n=m}^{b} x_{m, n} .
$$

Equation A.14 is expanded by using trigonometric summation rules and the previous formula.

$$
\begin{aligned}
&\left.\frac{\partial \phi^{i}}{\partial r_{i}}\right|_{r_{i}=R_{i}}= \sum_{j=1}^{K *} \sum_{m=1}^{\infty} \sum_{n=m}^{\infty}\left(\begin{array}{l}
n \\
m
\end{array}\right) \frac{m\left(R_{i}\right)^{m-1}}{\left(-P_{i j}\right)^{m-n}} A_{n j}\left[\cos \left(m \theta_{i}\right) \cos \left((n-m) \psi_{i j}\right)-\sin \left(m \theta_{i}\right) \sin \left((n-m) \psi_{i j}\right)\right]+ \\
& \sum_{j=1}^{K *} \sum_{m=1}^{\infty} \sum_{n=m}^{\infty}\left(\begin{array}{l}
n \\
m
\end{array}\right) \frac{m\left(R_{i}\right)^{m-1}}{\left(-P_{i j}\right)^{m-n}} B_{n j}\left[\sin \left(m \theta_{i}\right) \cos \left((n-m) \psi_{i j}\right)+\cos \left(m \theta_{i}\right) \sin \left((n-m) \psi_{i j}\right)\right]+ \\
& \sum_{j=1}^{K *} \sum_{m=1}^{\infty} \sum_{n=1}^{\infty}\left(\begin{array}{c}
-n \\
m
\end{array}\right) \frac{m\left(R_{i}\right)^{m-1}}{\left(-P_{i j}\right)^{n+m}} C_{n j}\left[\cos \left(m \theta_{i}\right) \cos \left((n+m) \psi_{i j}\right)+\sin \left(m \theta_{i}\right) \sin \left((n+m) \psi_{i j}\right)\right]+ \\
& \sum_{j=1}^{K *} \sum_{m=1}^{\infty} \sum_{n=1}^{\infty}\left(\begin{array}{c}
-n \\
m
\end{array}\right) \frac{m\left(R_{i}\right)^{m-1}}{\left(-P_{i j}\right)^{n+m}} D_{n j}\left[-\sin \left(m \theta_{i}\right) \cos \left((n+m) \psi_{i j}\right)+\cos \left(m \theta_{i}\right) \sin \left((n+m) \psi_{i j}\right)\right]+ \\
& \sum_{n=1}^{\infty} n R_{i}^{n-1}\left(A_{n i} \cos \left(n \theta_{i}\right)+B_{n i} \sin \left(n \theta_{i}\right)\right)-n R_{i}^{-n-1}\left(C_{n i} \cos \left(n \theta_{i}\right)+D_{n i} \sin \left(n \theta_{i}\right)\right)
\end{aligned}
$$

By using the independence of the trigonometric functions and the boundary condition in Equation A.12, the following sets of Equations are found:

-the coefficients of $\cos \left(m \theta_{i}\right)$ :

$$
\begin{array}{r}
\sum_{j=1}^{K *} \sum_{n=m}^{\infty}\left(\begin{array}{c}
n \\
m
\end{array}\right) \frac{m\left(R_{i}\right)^{m-1}}{\left(-P_{i j}\right)^{m-n}}\left[A_{n j} \cos \left((n-m) \psi_{i j}\right)+B_{n j} \sin \left((n-m) \psi_{i j}\right)\right]+ \\
\sum_{j=1}^{K *} \sum_{n=1}^{\infty}\left(\begin{array}{c}
-n \\
m
\end{array}\right) \frac{m\left(R_{i}\right)^{m-1}}{\left(-P_{i j}\right)^{n+m}}\left[C_{n j} \cos \left((n+m) \psi_{i j}\right)+D_{n j} \sin \left((n+m) \psi_{i j}\right)\right]+ \\
m R_{i}^{m-1} A_{m i}-m R_{i}^{-m-1} C_{m i}=\delta_{1 m} \frac{\partial u_{i}}{\partial t}
\end{array}
$$


-the coefficients of $\sin \left(m \theta_{i}\right)$ :

$$
\begin{array}{r}
\sum_{j=1}^{K *} \sum_{n=m}^{\infty}\left(\begin{array}{c}
n \\
m
\end{array}\right) \frac{m\left(R_{i}\right)^{m-1}}{\left(-P_{i j}\right)^{m-n}}\left[-A_{n j} \sin \left((n-m) \psi_{i j}\right)+B_{n j} \cos \left((n-m) \psi_{i j}\right)\right]+ \\
\sum_{j=1}^{K *} \sum_{n=1}^{\infty}\left(\begin{array}{c}
-n \\
m
\end{array}\right) \frac{m\left(R_{i}\right)^{m-1}}{\left(-P_{i j}\right)^{n+m}}\left[C_{n j} \sin \left((n+m) \psi_{i j}\right)-D_{n j} \cos \left((n+m) \psi_{i j}\right)\right]+ \\
m R_{i}^{m-1} B_{m i}-m R_{i}^{-m-1} D_{m i}=\delta_{1 m} \frac{\partial v_{i}}{\partial t}
\end{array}
$$

The previous two equations are valid for $m=1 . . \infty$ and $i=1 . . K$. The A,B,C,D-constants are linear combinations of $\frac{\partial u_{i}}{\partial t}$ and $\frac{\partial v_{i}}{\partial t}$ :

$$
\begin{gathered}
A_{m i}=R_{i}^{-m+1} \sum_{l=1}^{K} \alpha_{m i l} \frac{\partial u_{l}}{\partial t}+a_{m i l} \frac{\partial v_{l}}{\partial t}, B_{m i}=R_{i}^{-m+1} \sum_{l=1}^{K} \beta_{m i l} \frac{\partial u_{l}}{\partial t}+b_{m i l} \frac{\partial v_{l}}{\partial t} \\
C_{m i}=R_{i}^{m+1} \sum_{l=1}^{K} \gamma_{m i l} \frac{\partial u_{l}}{\partial t}+c_{m i l} \frac{\partial v_{l}}{\partial t}, D_{m i}=R_{i}^{m+1} \sum_{l=1}^{K} \delta_{m i l} \frac{\partial u_{l}}{\partial t}+d_{m i l} \frac{\partial v_{l}}{\partial t} .
\end{gathered}
$$

After substitution of those relations in Equation A.17 and A.18 and using the independence of $u_{l}$ and $v_{l}$, the following (final) sets of equations are obtained.

$$
\begin{aligned}
& \sum_{j=1}^{K *}\left(\sum_{n=m}^{\infty} f_{1, n m}\left[\alpha_{n j l} \cos _{1, n m}+\beta_{n j l} \sin _{1, n m}\right]+\sum_{n=1}^{\infty} f_{2, n m}\left[\gamma_{n j l} \cos _{2, n m}+\delta_{n j l} \sin _{2, n m}\right]\right)+m\left(\alpha_{m i l}-\gamma_{m i l}\right)=\delta_{1 m} \delta_{i l}, \\
& \sum_{j=1}^{K *}\left(\sum_{n=m}^{\infty} f_{1, n m}\left[a_{n j l} \cos _{1, n m}+b_{n j l} \sin _{1, n m}\right]+\sum_{n=1}^{\infty} f_{2, n m}\left[c_{n j l} \cos _{2, n m}+d_{n j l} \sin _{2, n m}\right]\right)+m\left(a_{m i l}-c_{m i l}\right)=0, \\
& \sum_{j=1}^{K *}\left(\sum_{n=m}^{\infty} f_{1, n m}\left[-\alpha_{n j l} \sin _{1, n m}+\beta_{n j l} \cos _{1, n m}\right]+\sum_{n=1}^{\infty} f_{2, n m}\left[\gamma_{n j l} \sin _{2, n m}-\delta_{n j l} \cos _{2, n m}\right]\right)+m\left(\beta_{m i l}-\delta_{m i l}\right)=0, \\
& \sum_{j=1}^{K *}\left(\sum_{n=m}^{\infty} f_{1, n m}\left[-a_{n j l} \sin _{1, n m}+b_{n j l} \cos _{1, n m}\right]+\sum_{n=1}^{\infty} f_{2, n m}\left[c_{n j l} \sin _{2, n m}-d_{n j l} \cos _{2, n m}\right]\right)+m\left(b_{m i l}-d_{m i l}\right)=\delta_{1 m} \delta_{i l},
\end{aligned}
$$

with :

$$
\begin{aligned}
& f_{1, n m}=\left(\begin{array}{l}
n \\
m
\end{array}\right) \frac{m\left(R_{i}\right)^{m-n}}{\left(-P_{i j}\right)^{m-n}}, f_{2, n m}=\left(\begin{array}{c}
-n \\
m
\end{array}\right) \frac{m\left(R_{i}\right)^{m+n}}{\left(-P_{i j}\right)^{n+m}}, \\
& \cos _{1, n m}=\cos \left((n-m) \psi_{i j}\right), \sin _{1, n m}=\sin \left((n-m) \psi_{i j}\right), \\
& \cos _{2, n m}=\cos \left((n+m) \psi_{i j}\right), \sin _{2, n m}=\sin \left((n+m) \psi_{i j}\right) .
\end{aligned}
$$

In this expression, $m$ ranges from 1 to infinity and $i$ and $l$ from 1 to $K$. If the series expansion is truncated at $N$, this results in $4 N K^{2}$ equations (with $K=7$ and $N=11,2156$ equations) in $8 N K^{2}$ variables. The remaining necessary equations come from the boundary condition of the exterior containment of the bundle.

\section{Appendix B. Inviscid normal hydrodynamic force} by:

According to the derivation of Lighthill (1960), the inviscid normal hydrodynamic force for slender bodies is given

$$
F_{N I i}^{x}=-\int_{0}^{2 \pi} \rho_{f} \frac{D \phi_{i}\left(r=R_{i}\right)}{D t} R_{i} \cos \theta_{i} \mathrm{~d} \theta_{i}
$$


Upon substitution of Equation A.4 in B.1, the lateral inviscid force becomes

$$
F_{N I i}^{x}=-\int_{0}^{2 \pi} \rho_{f} R_{i} \cos \left(\theta_{i}\right) \sum_{n=1}^{\infty} \frac{\mathrm{D} A_{n i}}{\mathrm{D} t} R_{i}^{n} \cos \left(n \theta_{i}\right)+\frac{\mathrm{D} B_{n i}}{\mathrm{D} t} R_{i}^{n} \sin \left(n \theta_{i}\right)+\frac{\mathrm{D} C_{n i}}{\mathrm{D} t} R_{i}^{-n} \cos \left(n \theta_{i}\right)+\frac{\mathrm{D} D_{n i}}{\mathrm{D} t} R_{i}^{-n} \sin \left(n \theta_{i}\right) \mathrm{d} \theta_{i}
$$

Using the symmetry properties of the involved products of trigonometric terms, the previous expression simplifies to

$$
F_{N I i}^{x}=-\int_{0}^{2 \pi} \rho R_{i} \cos \left(\theta_{i}\right)\left(\frac{\mathrm{D} A_{1 i}}{\mathrm{D} t} R_{i} \cos \left(\theta_{i}\right)+\frac{\mathrm{D} C_{1 i}}{\mathrm{D} t} R_{i}^{-1} \cos \left(\theta_{i}\right)\right) \mathrm{d} \theta_{i} .
$$

Substituting Equation A.19 (with material derivative) and evaluating the integral results in:

$$
F_{N I i}^{x}=-\rho_{f} \pi R_{i}^{2} \sum_{l=1}^{K}\left(\left(\alpha_{1 i l}+\gamma_{1 i l}\right) \frac{\mathrm{D}^{2} u_{l}}{\mathrm{D} t^{2}}+\left(a_{1 i l}+c_{1 i l}\right) \frac{\mathrm{D}^{2} v_{l}}{\mathrm{D} t^{2}}\right)
$$

In the y-direction, using a similar derivation, the normal force becomes:

$$
F_{N I i}^{y}=-\rho_{f} \pi R_{i}^{2} \sum_{l=1}^{K}\left(\left(\beta_{1 i l}+\delta_{1 i l}\right) \frac{\mathrm{D}^{2} u_{l}}{\mathrm{D} t^{2}}+\left(b_{1 i l}+d_{1 i l}\right) \frac{\mathrm{D}^{2} v_{l}}{\mathrm{D} t^{2}}\right)
$$

\section{Appendix C. Viscous normal hydrodynamic motion}

In section 2.1 the normal velocity perturbation due to the movement of neighboring cylinders is required. This can be computed from the potential

$$
\phi^{i}=\sum_{l=1}^{K *} \phi_{l}^{i}+\phi_{i},
$$

which is the potential centered around cylinder $i$. The average normal flow velocity results from following integration

$$
\begin{aligned}
& V_{x i}=\frac{1}{2 \pi} \int_{0}^{2 \pi} \nabla \phi^{i} \cdot 1_{x} \mathrm{~d} \theta_{i}=\frac{1}{2 \pi} \int_{0}^{2 \pi}-\frac{1}{r_{i}} \frac{\partial \phi^{i}}{\partial \theta_{i}} \sin \left(\theta_{i}\right)+\frac{\partial \phi^{i}}{\partial r_{i}} \cos \left(\theta_{i}\right) \mathrm{d} \theta_{i} \\
& V_{y i}=\frac{1}{2 \pi} \int_{0}^{2 \pi} \nabla \phi^{i} \cdot 1_{y} \mathrm{~d} \theta_{i}=\frac{1}{2 \pi} \int_{0}^{2 \pi} \frac{1}{r_{i}} \frac{\partial \phi^{i}}{\partial \theta_{i}} \cos \left(\theta_{i}\right)+\frac{\partial \phi^{i}}{\partial r_{i}} \sin \left(\theta_{i}\right) \mathrm{d} \theta_{i},
\end{aligned}
$$

where the integrand is evaluated at the cylinder's wall $\left(r_{i}=R_{i}\right)$. The partial derivative of this potential with respect to the polar angle is

$$
\begin{aligned}
\frac{\partial \phi^{i}\left(r_{i}, \theta_{i}\right)}{\partial \theta_{i}} & =\sum_{l=1}^{K *} \sum_{n=1}^{\infty} \sum_{m=0}^{n}\left(\begin{array}{l}
n \\
m
\end{array}\right) \frac{\left(r_{i}\right)^{m}}{\left(-P_{i l}\right)^{m-n}}\left(-m A_{n l} \sin \left(m \theta_{i}+(n-m) \psi_{i l}\right)+m B_{n l} \cos \left(m \theta_{i}+(n-m) \psi_{i l}\right)\right) \\
& +\sum_{l=1}^{K *} \sum_{n=1}^{\infty} \sum_{m=0}^{\infty}\left(\begin{array}{c}
-n \\
m
\end{array}\right) \frac{\left(r_{i}\right)^{m}}{\left(-P_{i l}\right)^{n+m}}\left(-m C_{n l} \sin \left(m \theta_{i}-(n+m) \psi_{i l}\right)-m D_{n l} \cos \left(m \theta_{i}-(n+m) \psi_{i l}\right)\right) \\
& +\sum_{m=1}^{\infty}-m A_{m i} r_{i}^{m} \sin \left(m \theta_{i}\right)+m B_{m i} r_{i}^{m} \cos \left(m \theta_{i}\right)-m C_{m i} r_{i}^{-m} \sin \left(m \theta_{i}\right)+m D_{m i} r_{i}^{-m} \cos \left(m \theta_{i}\right) .
\end{aligned}
$$

In integral C. 2 the only terms that will not result in zero are those in $\cos \left(\theta_{i}\right)^{2}$ and $\sin \left(\theta_{i}\right)^{2}$. The first term hence reduces to

$$
\begin{aligned}
& \sum_{l=1}^{K *} \sum_{n=1}^{\infty}\left(\begin{array}{c}
n \\
1
\end{array}\right) \frac{1}{\left(-P_{i l}\right)^{1-n}}\left(A_{n l} \cos \left((n-1) \psi_{i l}\right)+B_{n l} \sin \left((n-1) \psi_{i l}\right)\right) \sin \left(\theta_{i}\right) \\
& +\sum_{l=1}^{K *} \sum_{n=1}^{\infty}\left(\begin{array}{c}
-n \\
1
\end{array}\right) \frac{1}{\left(-P_{i l}\right)^{n+1}}\left(C_{n l} \cos \left((n+1) \psi_{i l}\right)+D_{n l} \sin \left(-(n+1) \psi_{i l}\right)\right) \sin \left(\theta_{i}\right) \\
& +\left(A_{1 i}+R_{i}^{-2} C_{1 i}\right) \sin \left(\theta_{i}\right) .
\end{aligned}
$$


The second term similarly reduces to

$$
\begin{aligned}
& \sum_{j=1}^{K *} \sum_{n=1}^{\infty}\left(\begin{array}{c}
n \\
1
\end{array}\right) \frac{1}{\left(-P_{i j}\right)^{1-n}}\left[A_{n j} \cos \left((n-1) \psi_{i j}\right)+B_{n j} \sin \left((n-1) \psi_{i j}\right)\right] \cos \left(\theta_{i}\right) \\
& +\sum_{j=1}^{K *} \sum_{n=1}^{\infty}\left(\begin{array}{c}
-n \\
1
\end{array}\right) \frac{1}{\left(-P_{i j}\right)^{n+1}}\left[C_{n j} \cos \left((n+1) \psi_{i j}\right)+D_{n j} \sin \left((n+1) \psi_{i j}\right)\right] \cos \left(\theta_{i}\right) \\
& +\left(A_{1 i}-R_{i}^{-2} C_{1 i}\right) \cos \left(\theta_{i}\right) .
\end{aligned}
$$

Substituting Equation A.19 (with material derivative) and evaluating the integral results in

$$
\begin{aligned}
V_{x i}= & \sum_{j=1}^{K *} \sum_{n=1}^{\infty} \sum_{l=1}^{K} n \frac{R_{j}^{-n+1}}{\left(-P_{i j}\right)^{1-n}}\left(\left[\alpha_{n j l} \frac{\mathrm{D} u_{l}}{\mathrm{D} t}+a_{n j l} \frac{\mathrm{D} v_{l}}{\mathrm{D} t}\right] \cos \left((n-1) \psi_{i j}\right)+\left[\beta_{n j l} \frac{\mathrm{D} u_{l}}{\mathrm{D} t}+b_{n j l} \frac{\mathrm{D} v_{l}}{\mathrm{D} t}\right] \sin \left((n-1) \psi_{i j}\right)\right) \\
& +\sum_{j=1}^{K *} \sum_{n=1}^{\infty} \sum_{l=1}^{K} n \frac{R_{i}^{n+1}}{\left(-P_{i j}\right)^{n+1}}\left(\left[-\gamma_{n j l} \frac{\mathrm{D} u_{l}}{\mathrm{D} t}-c_{n j l} \frac{\mathrm{D} v_{l}}{\mathrm{D} t}\right] \cos \left((n+1) \psi_{i j}\right)+\left[-\delta_{m i l} \frac{\mathrm{D} u_{l}}{\mathrm{D} t}-d_{m i l} \frac{\mathrm{D} v_{l}}{\mathrm{D} t}\right] \sin \left((n+1) \psi_{i j}\right)\right) \\
& +\sum_{l=1}^{K}\left[\alpha_{1 i l} \frac{\mathrm{D} u_{l}}{\mathrm{D} t}+a_{1 i l} \frac{\mathrm{D} v_{l}}{\mathrm{D} t}\right]
\end{aligned}
$$

The induced velocity in the y-direction is similarly:

$$
\begin{aligned}
V_{y i}= & \sum_{j=1}^{K *} \sum_{n=1}^{\infty} \sum_{l=1}^{K} n \frac{R_{j}^{-n+1}}{\left(-P_{i j}\right)^{1-n}}\left(\left[-\alpha_{n j l} \frac{\mathrm{D} u_{l}}{\mathrm{D} t}-a_{n j l} \frac{\mathrm{D} v_{l}}{\mathrm{D} t}\right] \sin \left((n-1) \psi_{i j}\right)+\left[\beta_{n j l} \frac{\mathrm{D} u_{l}}{\mathrm{D} t}+b_{n j l} \frac{\mathrm{D} v_{l}}{\mathrm{D} t}\right] \cos \left((n-1) \psi_{i j}\right)\right) \\
& +\sum_{j=1}^{K *} \sum_{n=1}^{\infty} \sum_{l=1}^{K} n \frac{R_{i}^{n+1}}{\left(-P_{i j}\right)^{n+1}}\left(\left[-\gamma_{n j l} \frac{\mathrm{D} u_{l}}{\mathrm{D} t}-c_{n j l} \frac{\mathrm{D} v_{l}}{\mathrm{D} t}\right] \sin \left((n+1) \psi_{i j}\right)+\left[\delta_{m i l} \frac{\mathrm{D} u_{l}}{\mathrm{D} t}+d_{m i l} \frac{\mathrm{D} v_{l}}{\mathrm{D} t}\right] \cos \left((n+1) \psi_{i j}\right)\right) \\
& +\sum_{l=1}^{K}\left[\beta_{1 i l} \frac{\mathrm{D} u_{l}}{\mathrm{D} t}+b_{1 i l} \frac{\mathrm{D} v_{l}}{\mathrm{D} t}\right]
\end{aligned}
$$

The coefficients can be found from Equation A.20.

Note that the derivation in this section differs substantially from Païdoussis and Suss (1977). In contrast to earlier work, the total flow potential is used. In Païdoussis and Suss (1977), the potential due to cylinder $i$ itself is omitted and Equation A.20 is adapted similarly. The diagonal elements of the velocity matrix are set to one. This means that if a cylinder was moving in the transverse direction with a speed $v$, the average relative flow speed over the cylinder would be $-v$. However, in a confined area, such as a rod bundle, the fluid is squeezed through gaps and consequently the relative flow speed increases. The increase due to this confinement effect is present in the total potential used in this part. The diagonal elements of the velocity matrix are in this work the sum of the direct velocity of the cylinder and the induced velocity by the presence of neighboring structures. It was verified that the induced velocity of solitary cylinders (in rod bundles with a very large pitch-over-diameter ratio) effectively reduces to zero with the present description.

A second difference is that material derivatives are used in this article, while in Païdoussis and Suss (1977) time derivatives are used. By using material derivatives the relative flow velocity induced by the bending or tilting of a neighboring cylinder is included. The present description of the transverse velocities should hence be more complete and accurate. A second advantage is that only one potential is required and Equation A.20 needs to be solved only once.

\section{References}

Chen, S. S., 1987. Flow-induced vibration of circular cylindrical structures. Hemisphere Pub. Corp. 
Cheng, S. K., Todreas, N. E., 1986. Hydrodynamic models and correlations for bare and wire-wrapped hexagonal rod bundles - bundle friction factors, subchannel friction factors and mixing parameters. Nuclear Engineering and Design 92 (2), $227-251$.

de Langre, E., Païdoussis, M. P., Doaré, O., Modarres-Sadeghi, Y., 2007. Flutter of long flexible cylinders in axial flow. Journal of Fluid Mechanics 571, 371-389.

De Ridder, J., Degroote, J., Van Tichelen, K., Schuurmans, P., Vierendeels, J., 2013. Modal characteristics of a flexible cylinder in turbulent axial flow from numerical simulations. Journal of Fluids and Structures 43, 110-123.

De Ridder, J., Doaré, O., Degroote, J., Van Tichelen, K., Schuurmans, P., Vierendeels, J., 2015. Simulating the fluid forces and fluid-elastic instabilities of a clamped-clamped cylinder in turbulent axial flow. Journal of Fluids and Structures 55, 139-154.

Degroote, J., Bathe, K. J., Vierendeels, J., 2009. Performance of a new partitioned procedure versus a monolithic procedure in fluid-structure interaction. Computers and Structures 87 (11-12), 793-801.

Divaret, L., Cadot, O., Moussou, P., Doaré, O., 2014. Normal forces exerted upon a long cylinder oscillating in an axial flow. Journal of Fluid Mechanics 752, 649-669.

Ersdal, S., Faltinsen, O. M., 2006. Normal forces on cylinders in near-axial flow. Journal of Fluids and Structures 22 (8), $1057-1077$.

Hoerner, S., 1965. Fluid-dynamic drag: practical information on aerodynamic drag and hydrodynamic resistance. Hoerner Fluid Dynamics

Hooper, J. D., Rehme, K., 1984. Large-scale structural effects in developed turbulent-flow through closely-spaced rod arrays. Journal of Fluid Mechanics 145, 305-337.

Jamal, A., Païdoussis, M. P., Mongeau, L. G., 2014. Linear and nonlinear dynamics of cantilevered cylinders in axial flow. In: ASME 2014 Pressure Vessels and Piping Conference. American Society of Mechanical Engineers, pp. V004T04A027-V004T04A027.

Kheiri, M., Païdoussis, M. P., 2015. Dynamics and stability of a flexible pinned-free cylinder in axial flow. Journal of Fluids and Structures 55, 204-217.

Lighthill, M. J., 1960. Note on the swimming of slender fish. Journal of Fluid Mechanics 9 (2), 305-317.

Lin, W. H., 1987. Hydrodynamic-forces on multiple circular-cylinders oscillating in a viscous incompressible fluid. Zeitschrift Fur Angewandte Mathematik Und Mechanik 67 (10), 487-501.

Lopes, J. L., Païdoussis, M. P., Semler, C., 2002. Linear and nonlinear dynamics of cantilevered cylinders in axial flow. Part 2: The equations of motion. Journal of Fluids and Structures 16 (6), 715-737.

Menter, F. R., 1994. 2-equation eddy-viscosity turbulence models for engineering applications. AIAA Journal 32 (8), $1598-1605$.

Meyer, L., Rehme, K., 1994. Large-scale turbulence phenomena in compound rectangular channels. Experimental Thermal and Fluid Science 8 (4), 286-304.

Modarres-Sadeghi, Y., Païdoussis, M. P., Semler, C., 2007. The nonlinear behaviour of a slender flexible cylinder pinned or clamped at both ends and subjected to axial flow. Computers and Structures 85 (11-14), 1121-1133.

Modarres-Sadeghi, Y., Païdoussis, M. P., Semler, C., Grinevich, E., 2008. Experiments on vertical slender flexible cylinders clamped at both ends and subjected to axial flow. Philosophical Transactions of the Royal Society A-Mathematical Physical and Engineering Sciences 366 (1868), 1275-1296.

Möller, S. V., 1991. On phenomena of turbulent-flow through rod bundles. Experimental Thermal and Fluid Science 4 (1), $25-35$.

Morison, J., O’ Brien, M. P., Johnson, J., Schaaf, S. A., 1950. The forces exerted by surface waves on piles. Petroleum transactions, AIME 189, 149-157.

Païdoussis, M. P., 1973. Dynamics of cylindrical structures subjected to axial flow. Journal of Sound and Vibration 29 (3), $365-385$.

Païdoussis, M. P., 1979. Dynamics of clusters of flexible cylinders in axial-flow - theory and experiments. Journal of Sound and Vibration 65 (3), 391-417.

Païdoussis, M. P., Grinevich, E., Adamovic, D., Semler, C., 2002. Linear and nonlinear dynamics of cantilevered cylinders in axial flow. Part 1: Physical dynamics. Journal of Fluids and Structures 16 (6), 691-713.

Païdoussis, M. P., Suss, S., 1977. Stability of a cluster of flexible cylinders in bounded axial-flow. Journal of Applied Mechanics-Transactions of the Asme 44 (3), 401-408.

Rehme, K., 1992. The structure of turbulence in rod bundles and the implications on natural mixing between the subchannels. International Journal of Heat and Mass Transfer 35 (2), 567-581.

Rinaldi, S., Païdoussis, M. P., 2012. Theory and experiments on the dynamics of a free-clamped cylinder in confined axial air-flow. Journal of Fluids and Structures 28, 167-179.

Sakuma, Y., Païdoussis, M. P., Price, S. J., 2008. Dynamics of trains and train-like articulated systems travelling in confined fluid-Part 1: Modelling and basic dynamics. Journal of Fluids and Structures 24 (7), 932-953.

Semler, C., Lopes, J. L., Augu, N., Païdoussis, M. P., 2002. Linear and nonlinear dynamics of cantilevered cylinders in axial flow. part 3: Nonlinear dynamics. Journal of Fluids and Structures 16 (6), 739-759.

Taylor, G., 1952. Analysis of the swimming of long and narrow animals. Proceedings of the Royal Society Series 214 (1117), 158-183.

Triantafyllou, M. S., 1985. The dynamics of translating cables. Journal of Sound and Vibration 103 (2), $171-182$. 